\title{
Réseaux des liens matrimoniaux
}

Le choix du conjoint dans une commune toscane au $\mathrm{xx}^{\mathrm{e}}$ siècle

\section{Silvia Sinibaldi}

\section{OpenEdition}

1 Journals

Édition électronique

URL : http://journals.openedition.org/lhomme/21830

DOI : $10.4000 /$ /homme.21830

ISSN : 1953-8103

Éditeur

Éditions de l'EHESS

\section{Édition imprimée}

Date de publication : 1 février 2007

Pagination : 41-73

ISSN : 0439-4216

\section{Référence électronique}

Silvia Sinibaldi, « Réseaux des liens matrimoniaux », L'Homme [En ligne], 181 | 2007, mis en ligne le 01 janvier 2009, consulté le 19 avril 2019. URL : http://journals.openedition.org//homme/21830 ; DOI : 10.4000//homme.21830

\section{(c) École des hautes études en sciences sociales}




\title{
Réseaux des liens matrimoniaux \\ Le choix du conjoint dans une commune toscane au $X x^{e}$ siècle
}

\section{Silvia Sinibaldi}

\begin{abstract}
Des communications transversales entre lignes différenciées brouillent les arbres généalogiques. [...] Le rhizome est une antigénéalogie. [...] Un rhizome ne commence et n'aboutit pas, il est toujours au milieu, entre les choses, inter-être, intermezzo. L'arbre est filiation, mais le rhizome est alliance, uniquement d'alliance. L'arbre impose le verbe "être", mais le rhizome a pour tissu la conjonction "et... et... et..." Il y a dans cette conjonction assez de force pour secourir et déraciner le verbe être. Gilles Deleuze \& Félix Guattari $(1980: 18,36)$.
\end{abstract}

L

-UCignano in VAl di Chiana est un bourg du sud de la Toscane ${ }^{1}$ (Italie) comprenant 3468 habitants en 2001. De 1999 à 2003, il a fait l'objet d'une recherche historique, anthropologique et démographique concernant la nuptialité, les formes de famille, le patrimoine, les réseaux de parenté, les noms de famille.

L'essai propose un examen des mécanismes de sélection du conjoint par une analyse des caractéristiques des réseaux matrimoniaux de classes socioprofessionnelles spécifiques. Cet approfondissement de la configuration globale des réseaux du mariage d'une famille a nécessité la reconstruction généalogique des noms de famille (de 1650 à nos jours), le recueil de documents historiques relatifs à l'histoire familiale, ainsi que l'enregistrement systématique des sources orales. Un des objectifs de cette recherche est de démontrer l'utilité d'une méthode ethnographique composite, pouvant conjuguer des points de vue différents dans la substance: de la méthode statistique aux mémoires de famille, des configurations des réseaux à leur perception de la part des protagonistes. Les plans ethnographiques de traitement des données et des informations peuvent être résumés par les notions, désormais classiques, de "schémique ", "émique » et "éthique ».

1. Lucignano se trouve à $414 \mathrm{~m}$ d'altitude, dans le Val di Chiana, entre Sienne et Arezzo. D'un point de vue historique et artistique, le bourg représente un exemple caractéristique d'urbanisme médiéval par sa forme elliptique, composée de différents anneaux concentriques, en haut desquels on trouve le palais communal, l'église Saint-François et la collégiale.

Je voudrais exprimer mes remerciements les plus sincères à Mme Françoise Zonabend, pour la lecture de l'essai, et à M. Pascal Cristofoli (Laboratoire de démographie historique, Ehess), à qui je dois la réélaboration des données relationnelles pour l'utilisation du programme Pajek.

Article traduit par Sylvie Gaury et Silvia Sinibaldi. 
La construction schémique ressort de l'analyse de ce qu'on appelle les "sources écrites»: les archives informatisées des états civils, les actes de mariage, les documents familiaux, les archives des cimetières, les reconstructions généalogiques, les documents paroissiaux, les recensements. Par l'élaboration statistique et l'application de la théorie des graphes aux données relationnelles, le point de vue schémique cherche à pénétrer les données globales et à communiquer les observations effectuées dans une forme graphique. Jacques Bertin (1999) parlerait de "sémiologie graphique » : un "langage » destiné à l'œil, à la vue, expression rationnelle du monde des images.

La vision émique est le point de vue local inhérent au choix du conjoint, la perception individuelle des liens. Ce choix du conjoint, cependant, a été analysé avec une méthode biographique par collectivité : non pas à partir des histoires de vie mais des mémoires de famille. La mémoire de famille est en effet l'un des «biens immatériels " les plus significatifs de la personne; son importance provient de ce qu'elle est une mémoire fondatrice (Halbwachs 1994 [1925]). On définit comme fondatrices les mémoires qui demeurent intègres et qui gardent la même charge d'affectivité tout au long des générations; en ce sens, elles participent à la construction des identités, des habitus, des représentations, et cela est clairement visible dans le discours émique.

La réflexion éthique représente, en anthropologie, l'appareil théorique des notions et des paradigmes par lesquels sont interprétées et comparées les données recueillies; en particulier, les notions de graphe et de réseau, d'aire matrimoniale, de choix, apparaissent plus ou moins fréquemment dans le débat interdisciplinaire sur les critères de sélection du conjoint. La notion de choix s'oppose à celle de panmixie, parce qu'elle implique une conception non aléatoire des unions matrimoniales, et fait référence au concept de "valeur matrimoniale» des potentiels conjoints. La valeur matrimoniale se compose de la "valeur» des biens matériels (salaire, héritage, propriétés, etc.) et symboliques (beauté, instruction, milieu familial, etc.) ${ }^{2}$ de la personne. La combinaison multiple de telles valeurs produit la variété des candidatures et, par conséquent, le choix. Il n'est pas possible d'approfondir dans cet essai les implications économiques et philosophiques de la notion de valeur matrimoniale (Sinibaldi 2002-2003, 2004) ; le concept est employé ici essentiellement pour mettre en évidence des modalités de sélection du conjoint et pour explorer le poids social, économique et symbolique des réseaux matrimoniaux construits par les familles au cours des générations. 
La représentation schémique, la vision émique et la réflexion éthique ont été décomposées en trois niveaux de traitement des données, pour favoriser l'exposé de la question. En ce qui concerne la méthode, cependant, elles désignent des plans cognitifs qui interagissent: pendant la recherche, les représentations schémiques ont été montrées aux interviewés afin de les comparer avec leur perception du réseau parental d'appartenance, de même que la définition éthique du réseau a été lue à la lumière de la vision émique de lien.

\section{Les aires matrimoniales}

En 2002, les actes de mariages concernant Lucignano du $1^{\text {er }}$ janvier 1900 au 31 décembre 2000 (pour un total de 4290, soit de 8580 conjoints) ont été transcrits dans une base de données.

Cette source conserve donc toutes les informations sur les mariages qui ont été célébrés par les résidents au cours du XX $\mathrm{X}^{\mathrm{e}}$ siècle ${ }^{3}$ : date et lieu de la cérémonie, rite, état civil des époux (nom et prénom, âge, profession), commune d'origine et commune de résidence au moment du mariage, paternité et maternité 4 , témoins, observations du curé ou de l'officier de l'état civil ${ }^{5}$. Les données associées, reportées dans d'autres tables, dérivent de l'élaboration des entrées "date du mariage ", " commune de naissance " et "commune de résidence"; en revanche, les informations qui concernent le nom, le prénom, la paternité et la maternité des époux ont été employées de manière dissociée pour la construction des graphes (cf. infra figures $5,6,8$ et 10 ).

Les données relatives au lieu de naissance et de résidence des époux sont élaborées en suivant un modèle d'aire centrée ou d'aires matrimoniales. La notion d'aire matrimoniale permet d'explorer les mécanismes de sélection du conjoint par un principe spatial : la distance géographique entre les lieux de naissance et de résidence des candidats au mariage constitue

3. Les registres de la commune sont organisés en deux parties : les mariages civils célébrés dans la commune sont inscrits dans la partie I; les mariages religieux célébrés dans la commune se trouvent dans la partie IIA; la partie IIB comprend les mariages religieux célébrés dans une autre commune et la partie IIC les mariages civils célébrés dans une autre commune, ainsi que les mariages civils et religieux célébrés à l'étranger. Pour l'enregistrement de l'acte dans les parties IIB et IIC, un des deux époux doit résider à Lucignano.

4. Les données concernant la paternité et la maternité sont extrêmement précieuses pour ceux qui étudient la parenté. Malheureusement, une loi interdit depuis 1971 d'indiquer sur les actes de mariage, dans les dossiers de migration et dans d'autres documents d'état civil, les renseignements concernant la paternité et la maternité. Il n'est donc plus possible de reconstruire les lignes directe et collatérale avec les seuls actes de mariage.

5. Ces observations, lorsqu'on les trouve, indiquent le degré de consanguinité ou d'affinité entre les époux, ou bien soulignent que la célébration du mariage s'est faite par procuration, que l'époux était à la guerre et qu'il a obtenu une autorisation de la part de son commandant de régiment, etc. 
le principal facteur de structuration territoriale des réseaux matrimoniaux. Le modèle d'aire centrée s'applique à des populations infinies et continues. Dans une structure de réseau multicentrée, on fixe un centre (Lucignano) d'où se développe une représentation en cercles concentriques: une première aire territoriale $(\alpha 1)$ est constituée par les communes limitrophes (Sinalunga, Marciano, Rapolano, Foiano, Monte San Savino); une seconde aire territoriale $(\beta 1)$ comprend les communes limitrophes des limitrophes (Bucine, Castelnuovo Berardenga, Civitella in Val di Chiana, Trequanda, Castiglion Fiorentino, Asciano, Torrita, Cortona, Arezzo); une troisième aire $(\gamma 1)$ englobe les communes restantes des provinces de Sienne et Arezzo (Solinas \& Grilli 2002 ; Sinibaldi 2002 et 2002$2003)^{6}$.

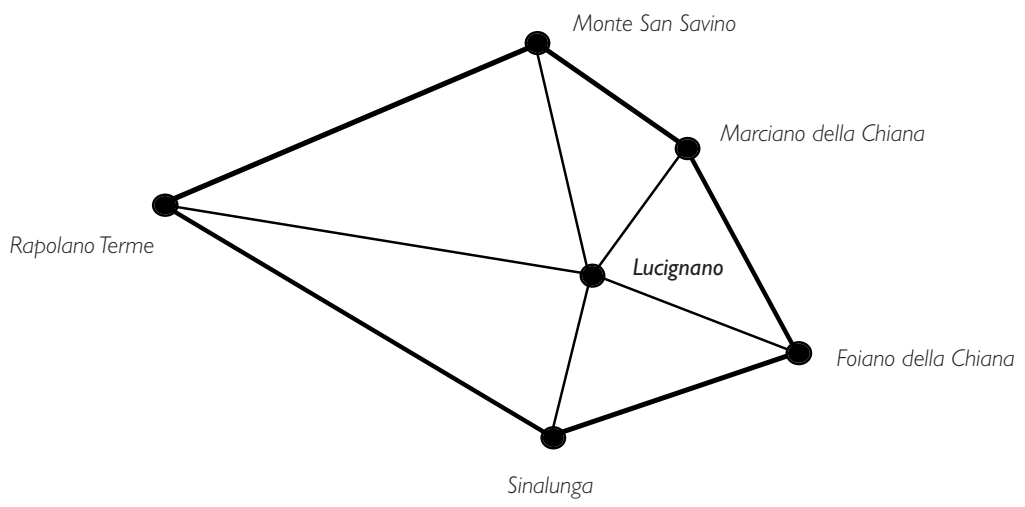

Fig. I Représentation de l'aire $\alpha$ |

Le principe sous-jacent est celui des ordres consécutifs de proximité spatiale qui apparaissent quand on fixe un centre à l'intérieur d'un réseau formé par l'intersection de plusieurs aires (chaque commune est centre d'une aire matrimoniale et marge des aires adjacentes), dans la perspective d'un continuum de peuplement. La configuration centrée, à la base d'une notion d'aire, ne peut être associée à un isolat démographique : le concept de centralité n'est qu'un artifice méthodologique employé pour tracer des

6. La recherche s'inscrivait dans la continuité d'une enquête précédente, statistique et démographique, menée par Pier Giorgio Solinas et Simonetta Grilli dans le sud de la Toscane sur les disquettes informatisées de l'état civil de la commune de Sinalunga et des communes limitrophes. En 1999, j’ai participé au projet de relevé démographique et j’ai élaboré les données sur la population des états civils informatisés des communes de Lucignano, Marciano, Monte San Savino, Foiano, Sinalunga et Rapolano Terme. Les données ont été regroupées dans deux bases de données, "Population" et "Mariages", de façon à former une aire de 41000 habitants avec, au centre, la commune de Lucignano. 
cercles concentriques de proximité spatiale. Bien sûr, en fixant un centre, la notion d'aire matrimoniale rappelle la notion de proximité, puisque l'aire matrimoniale se caractérise par la forte concentration des échanges matrimoniaux qui se produisent à l'intérieur de celle-ci et qui tendent à diminuer lorsque la distance augmente à partir du centre (Brunet, FauveChamoux \& Oris 1998). Si l'on adopte le principe des aires, il faut avant tout calculer les pourcentages d'endogamie et d'exogamie de la communecentre; de cette façon, il sera possible d'inspecter les caractéristiques matrimoniales du centre (Lucignano) de l'aire matrimoniale décrite cidessus.

Tableau I. Classes matrimoniales: naissance

(Source: Actes de mariage, 1900-2000)

$\begin{array}{lcc}\text { Naissance } & \text { Total } & \% \\ \text { Endogamie communale } & 915 & 21,33 \\ \text { Exogamie masculine } & 1018 & 23,73 \\ \text { Exogamie féminine } & 1096 & 25,55 \\ \text { Couples externes } & 1261 & 29,39 \\ \text { Total } & 4290 & 100 \\ \text { Total mariages exogamiques } & 2114 & 49,28\end{array}$

Tableau 2. Classes matrimoniales: résidence

(Source: Actes de mariage, 1900-2000)

$\begin{array}{lcc}\text { Résidence } & \text { Total } & \% \\ \text { Endogamie communale } & 1647 & 38,39 \\ \text { Exogamie masculine } & 1084 & 25,27 \\ \text { Exogamie féminine } & 1173 & 27,34 \\ \text { Couples externes } & 386 & 9,00 \\ \text { Total } & 4290 & 100 \\ \text { Total mariages exogamiques } & 2257 & 52,61\end{array}$

Endogamie communale: unions entre époux nés ou résidant dans la commune; exogamie masculine: mari né/résidant à Lucignano, femme née/résidant au-dehors; exogamie féminine : femme née/résidant à Lucignano et mari né/résidant au-dehors. Couples externes : les deux conjoints sont nés ou résident au-dehors, mais ils ont célébré leur mariage dans la commune de Lucignano. Les mariages entre époux nés au-dehors représentent près d'un tiers des unions; toutefois, cette classe nécessite des précisions ultérieures puisque dans les dernières décennies elle comprend également les habitants de Lucignano nés à Arezzo à cause des naissances en structure hospitalière (donc les mariages endogamiques). 
Quand on décompose les archives selon les trois classes principales, on observe que l'exogamie reste constante dans le passage de la naissance à la résidence, tandis que l'endogamie a presque doublé. L'endogamie de naissance représente plus de $20 \%$ des mariages, mais cette valeur est tributaire malheureusement du phénomène des naissances en structure hospitalière ${ }^{7}$ et s'élève à $38 \%$ dans l'analyse de la résidence des conjoints.

La méthode des aires matrimoniales et le regroupement des données par cohortes matrimoniales (date de célébration des mariages) permettent d'approfondir les directions préférentielles du choix du conjoint. De cette façon, il sera possible de mettre en évidence les changements survenus dans le marché matrimonial pendant le XXe siècle. Avec l'étude temporelle de la matrimonialité, il est possible d'isoler des comportements matrimoniaux différents pour les populations masculine et féminine, surtout en ce qui concerne certaines décennies.

Tableau 3. Endogamie et exogamie de naissance par cohortes matrimoniales: pourcentages sur les naissances pour chaque cohorte

(Source: Actes de mariage, 1900-2000)

\begin{tabular}{|c|c|c|c|c|c|c|}
\hline \multirow[t]{2}{*}{$\begin{array}{l}\text { Date du } \\
\text { mariage }\end{array}$} & \multicolumn{3}{|c|}{$\begin{array}{l}\text { Né(e) à Lucignano et marié(e) } \\
\text { à un(e) conjoint(e) né(e) à Lucignano }\end{array}$} & \multicolumn{3}{|c|}{$\begin{array}{l}\text { Né(e) à Lucignano et marié(e) } \\
\text { à un(e) conjoint(e) né(e) au-dehors }\end{array}$} \\
\hline & $\mathrm{H}$ & $\mathrm{F}$ & $\mathrm{T}$ & $\mathrm{H}$ & $\mathrm{F}$ & $\mathrm{T}$ \\
\hline $1900 / 1910$ & 58,95 & 49,70 & 53,93 & 41,05 & 50,30 & 46,07 \\
\hline $1911 / 1920$ & 53,74 & 49,36 & 51,45 & 46,26 & 50,64 & 48,55 \\
\hline $1921 / 1930$ & 52,77 & 56,25 & 54,45 & 47,23 & 43,75 & 45,55 \\
\hline $1931 / 1940$ & 54,04 & 55,95 & 54,98 & 45,96 & 44,05 & 45,02 \\
\hline $194|/| 950$ & 52,73 & 42,03 & 46,77 & 47,27 & 57,97 & 53,23 \\
\hline $1951 / 1960$ & 49,77 & 42,97 & 46,12 & 50,23 & 57,03 & 53,88 \\
\hline $1961 / 1970$ & 40,23 & 39,55 & 39,89 & 59,77 & 60,45 & 60,11 \\
\hline $1971 / 1980$ & 24,03 & 29,13 & 26,33 & 75,97 & 70,87 & 73,67 \\
\hline $1981 / 1990$ & 13,58 & 15,94 & 14,67 & 86,42 & 84,06 & 85,33 \\
\hline $199 \mid / 2000$ & 4,17 & 7,41 & 5,33 & 95,83 & 92,59 & 94,67 \\
\hline Total & 47,34 & 45,50 & 46,40 & 52,66 & 54,50 & 53,60 \\
\hline
\end{tabular}

7. Après 1965, avec les naissances en milieu hospitalier, les lieux de naissance des habitants de la commune sont Sinalunga, Foiano et surtout Arezzo. Les deux premières communes appartiennent à l'aire $\alpha 1$, la seconde à l'aire $\beta 1$. 
Date du mariage

$1900 / 1910$

$1911 / 1920$

$1921 / 1930$

$1931 / 1940$

$1941 / 1950$

$1951 / 1960$

$1961 / 1970$

1971/1980

$1981 / 1990$

$1991 / 2000$

Total
Né(e) à Lucignano et marié(e) à un(e) conjoint(e) né(e) en $\alpha$ l

$\begin{array}{lll}\mathrm{H} & \mathrm{F} & \mathrm{T}\end{array}$

$\begin{array}{lll}24,21 & 30,47 & 27,61 \\ 23,83 & 24,89 & 24,38 \\ 23,78 & 25,69 & 24,71 \\ 30,64 & 27,75 & 29,22 \\ 27,73 & 38,40 & 33,67 \\ 28,84 & 33,73 & 31,47 \\ 30,47 & 30,50 & 30,48 \\ 30,52 & 23,62 & 27,40 \\ 20,99 & 33,33 & 26,67 \\ 27,08 & 18,52 & 24,00\end{array}$

$26,79 \quad 29,84 \quad 28,34$
Né(e) à Lucignano et marié(e) à un(e) conjoint(e) né(e) en $\beta$ I
Né(e) à Lucignano et marié(e) à un(e) conjoint(e) né(e) en $\gamma \mathrm{l}$
Né(e) à Lucignano et marié(e) à un(e) conjoint(e) né(e) au-dehors de $\gamma \mathrm{l}$

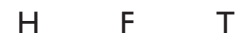

\section{$\begin{array}{lll}7,72 & 7,69 & 7,70\end{array}$}

$7,48 \quad 11,16 \quad 9,40$

$9,77 \quad 6,60 \quad 8,24$

$8,51 \quad 9,25 \quad 8,87$

$|0,9| \quad 5,80 \quad 8,06$

$9,30 \quad 9,25 \quad 9,27$

$8,62 \quad|4,13 \quad| 1,40$

$\begin{array}{lll}22,73 & 18,12 & 20,64\end{array}$

$30,86 \quad 26,09 \quad 28,67$

$33,33 \quad 40,74 \quad 36,00$

$\begin{array}{lll}11,54 & 10,34 & 10,93\end{array}$

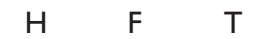

$\begin{array}{lll}3,16 & 7,99 & 5,78\end{array}$

$5,14 \quad 6,87 \quad 6,04$

$4,89 \quad 3,12 \quad 4,03$

$1,70 \quad 1,32 \quad 1,52$

$\begin{array}{lll}0,45 & 2,90 & 1,82\end{array}$

$\begin{array}{lll}1,86 & 3,21 & 2,58\end{array}$

$2,87 \quad 5,09 \quad 3,99$

$5,19 \quad \mid 1,81 \quad 8,19$

$\begin{array}{lll}7,41 & 7,25 & 7,33\end{array}$

$8,34 \quad 22,22 \quad 13,34$

$3,47 \quad 5,27 \quad 4,39$

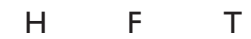

$5,96 \quad 4,15 \quad 4,98$

$\begin{array}{lll}9,81 & 7,72 & 8,73\end{array}$

$8,79 \quad 8,34 \quad 8,57$

$5,11 \quad 5,73 \quad 5,41$

$\begin{array}{lll}8,18 & 10,87 & 9,68\end{array}$

$\begin{array}{lll}10,23 & 10,84 & 10,56\end{array}$

$\begin{array}{lll}17,81 & 10,73 & 14,24\end{array}$

$\begin{array}{lll}17,53 & 17,32 & 17,44\end{array}$

$\begin{array}{lll}27,16 & 17,39 & 22,66\end{array}$

$27,08 \quad \mid 1,11 \quad 21,33$

$10,86 \quad 9,05 \quad 9,94$

L'analyse de l'endogamie de naissance montre que, jusqu'en 1970, les hommes sont fondamentalement plus endogames que les femmes: dans les deux décennies 1900-1910 et 1941-1950, on observe un écart d'environ dix points dans le comportement matrimonial des deux sexes. De 1900 à 1910 et de 1941 à 1950, les femmes de Lucignano, plus exogames, s'adressent principalement à l'aire $\alpha 1$ pour la recherche du conjoint. Dans les années 1941-1950, le marché matrimonial - qui enregistre à cette période un recul important du pourcentage d'unions endogamiques dans la population féminine - rétablit un équilibre non seulement pour les mariages exogamiques avec des hommes de l'aire $\alpha 1$, mais aussi pour ceux avec des candidats extérieurs à l'aire $\gamma 1$. À propos du déclin de l'endogamie masculine des deux décennies 1911-1920 et 1961-1970, on observe également, toujours dans le cadre de l'exogamie, une augmentation sensible des unions avec des femmes nées dans l'aire extérieure à $\gamma 1$.

Le déclin de l'endogamie communale au fil du temps n'est pas compensé par l'aire $\alpha 1$, ni par l'aire $\beta 1$ (dont les valeurs dans les dernières décennies sont surestimées par la présence d'Arezzo), mais par l'aire extérieure à $\gamma 1$ et, dans les années 1991-2000, aussi par l'aire $\gamma 1$. En effet, pendant cette décennie, $27 \%$ des hommes nés à Lucignano épousent une femme née au-dehors de $\gamma 1$, tandis que $22 \%$ des femmes de Lucignano épousent un homme de $\gamma 1$. Ici les choix matrimoniaux divergent nettement: les femmes, plus exogames, tendent cependant à rester dans les limites des provinces de Sienne et Arezzo; les hommes, plus endogames au cours du XXe siècle, ne se soustraient pas aux possibilités offertes par l'exogamie lointaine (les mariages au-dehors de $\gamma 1$ ). En revanche, 
une élaboration plus ponctuelle des données des aires $\alpha 1$ et $\beta 1$ montre que la commune de Rapolano, dans l'aire $\alpha 1$, a un poids fondamental dans l'exogamie féminine ${ }^{8}$, tandis que les communes de Cortona et de Castiglion Fiorentino (aire $\beta 1$ ) constituent des aires de choix privilégiées par la population masculine ${ }^{9}$.

Dans un souci de concision, on omettra d'utiliser d'autres analyses statistiques et démographiques : les tableaux qui précèdent ont essentiellement pour fonction de décrire la physionomie de l'aire matrimoniale de Lucignano. Les paragraphes suivants présentent un examen plus approfondi de l'endogamie, explorée à partir des caractéristiques de certains réseaux de parenté existant dans des zones spécifiques du territoire (bassins matrimoniaux), ainsi qu'une analyse de quelques directions préférentielles de choix du conjoint dans le cadre exogamique.

\section{Les sources orales : mémoires de famille}

\section{Les familles de petits propriétaires : les Dell'Angelo et les Marchi}

Dell'Angelo et Marchi ${ }^{10}$ sont deux noms de famille locaux qui, au XIXe siècle, étaient portés par de nombreuses personnes à Lucignano, mais qui, aujourd'hui, risquent de disparaître. À l'origine, ils font référence à deux familles de petits propriétaires - c'est-à-dire propriétaires exclusivement de la terre qu'ils travaillaient - qui s'étaient installées dans le hameau de Gorgone (paroisse de La Chiesina, commune de Lucignano).

Dans le Stato delle anime (l'État des âmes) de La Chiesina de 1841, le nom de Dell'Angelo apparaît plusieurs fois sur différentes propriétés. On y croise les familles de Pietro, Angiolo, Luigi et Antonio, tous propriétaires. Dans l'État des âmes de 1890, on trouve sept familles, ce qui représente un témoignage du développement, de la propagation, mais aussi de la segmentation de la famille au fil du temps. Dès le début du XIX siècle, le curé de l'église locale écrivait que les Dell'Angelo étaient « une famille très aisée travaillant pour elle».

Les Dell'Angelo cités dans les documents, porteurs donc du même patronyme, étaient les descendants de Giovanni, ancêtre apical, né en 1676. Ils habitaient sur les terres qu'ils cultivaient et dont ils revendiquaient

8. Au cours du XXe siècle, il y a 76 hommes nés à Rapolano qui épousent des femmes de Lucignano et 34 femmes de Rapolano qui épousent des hommes de Lucignano.

9. On constate que 44 épouses et 32 époux sont originaires de Cortona, tandis que 35 femmes et 28 hommes le sont de la commune de Castiglion Fiorentino.

10. En accord avec la loi sur la vie privée et avec les personnes interviewées, j’ai changé les noms de famille et de lieu, sauf le nom de la commune où s'est effectuée la recherche. D'éventuelles ressemblances entre des noms inventés et ceux existant réellement sont purement fortuites. 
la propriété; l'étendue des domaines agricoles (poderi) ${ }^{11}$ variait de trois à cinq hectares au début du $\mathrm{XX}^{\mathrm{e}}$ siècle : cela représentait la dimension moyenne d'un domaine par groupe domestique après des siècles de dévolutions patrimoniales entre les générations. Les témoins actuels parlent d'un patrimoine foncier ancien et consistant, successivement réparti entre les descendants de sexe masculin :

- An. : "Anciennement, les vieux [les ancêtres] avaient beaucoup d'argent, mais à mon époque, vous comprenez, mon défunt grand-père a eu beaucoup d'enfants et, à force de partager, on est arrivés à un point tel qu'on n'a presque plus rien..." (Interview de An. Dell'Angelo).

- C . : «Par exemple, nous, on a un proverbe qui dit: "Une famille fait de l'argent, une autre le garde, une autre le dépense : en trois générations ce que fait la première génération, la troisième le défait" " (Interview de A. Cor.).

À côté des Dell'Angelo, dans le hameau de Gorgone, habitaient les Marchi, descendants de Francesco, né à la fin de 1600 ; les Marchi étaient d'anciens métayers sur les terres de la Collégiale, mais, au XVIII siècle, ils devinrent propriétaires. De 1805 à 1814, le curé écrivait qu'ils étaient « des propriétaires de leurs terres très aisés ». Les descendants actuels disent que leur famille venait du nord:

Question : «Pouvez-vous me raconter l'histoire de la famille Marchi ?»

- A. : « Moi, je pense qu'ils venaient du nord de l'Italie, je ne sais pas exactement d'où, de Crémone ou de Parme. Ils ont dû venir ici au XVIII e siècle parce que je sais que ça fait longtemps. Ils ont acheté de la terre, cent hectares je crois, avec une grande belle maison, qui a été continuellement partagée. Moi, je suis le dernier des Mohicans, parce que je n'ai pas d'enfants" (Interview de A. Marchi).

Parmi les villageois, toutefois, il ne reste aujourd'hui que l'image d'une famille sédentaire :

— An.: «Moi, les Marchi, je les ai toujours connus là où ils sont morts... Là, à Gorgone, il y avait Settimio, il y avait untel et untel, et mon défunt père disait qu'il y en avait plein" (Interview de An. Dell'Angelo).

On mourait là où l'on naissait : c'était le destin des petits propriétaires, à la différence des métayers ${ }^{12}$ qui étaient plus exposés aux changements de

11. Le podere était un domaine agricole composé des terres arables avec ses bâtiments. Dans le cas des familles de petits propriétaires, le podere appartenait à la famille; dans le cas des familles de métayers, le podere (métairie) était intégré, avec d'autres métairies, dans le système de fattoria (propriété terrienne). La fattoria était une exploitation agricole constituée de plusieurs métairies administrées par un fattore (régisseur), appartenant au padrone (propriétaire). Le propriétaire stipulait avec les métayers le contrat de mezzadria (métayage) pour l'exploitation des métairies.

12. Le métayage était un contrat agricole, aujourd'hui aboli, suivant lequel le propriétaire des terres confiait au métayer la culture de celles-ci, selon un pacte de partage des bénéfices. Jusqu'au début des années 1960, il était assez répandu dans toute l'Italie centrale. 
métairies puisqu'ils ne possédaient pas la terre qu'ils travaillaient (Solinas 1987 et 1992). Cela produisait, parmi les habitants, l'identification du patronyme de la famille avec un lieu spécifique de la commune, où étaient nés et avaient grandi les descendants de l'ancêtre fondateur (en dialecte on dirait il ceppame, du mot ceppo, la « souche»).

La division patrimoniale entre les petits propriétaires suivait le modèle de la succession segmentaire agnatique et de l'héritage égalitaire sélectif (Augustins 1989 ; Pina Cabral 1989) ${ }^{13}$ : les biens immobiliers étaient transmis par le défunt aux fils en parties égales, les filles recevaient souvent une somme équivalente en argent, mais qui ne représentait jamais la valeur économique de la propriété dont avait hérité le fils (la legittima). Cela était aussi dû à la résidence patrivirilocale: les agnats restaient sur le domaine avec leurs familles respectives et, à la mort du chef de famille, ils en devenaient propriétaires; les filles, par le mariage, abandonnaient la maison paternelle. Toutefois, il arrivait qu'à la mort du père les fils décidaient d'appliquer la formule de la succession indivise agnatique et de l'héritage suspendu ${ }^{14}$ (qui donnait vie au modèle résidentiel de la frérèche ${ }^{15}$ ). Cette forme de dévolution restera en vigueur jusqu'aux années 1940 ; la fin de la vieille propriété paysanne et les transformations sociales et économiques des années 1950 et 1960 détermineront le passage de l'ancienne transmission des biens à la division égalitaire.

La femme qui était fille d'un cultivateur à son compte apportait son trousseau au moment du mariage; quand on lui donnait une certaine somme d'argent, en plus du trousseau, on parlait de dot. Si cette somme était élevée, il s'agissait souvent de la dot et de sa part d'héritage en tant que fille, c'est-àdire un héritage anticipé ${ }^{16}$. Il était possible, cependant, que la femme ait à son nom des biens fonciers - maisons et terres -, et cela pour l'une des raisons suivantes : l'absence de frères, l'impossibilité pour un père de convertir en argent la part qui revenait à sa fille, le choix d'un conjoint agriculteur.

13. Dans la succession segmentaire agnatique, chaque fils recevait le droit de fonder son propre groupe familial - et d'en devenir le chef de famille - indépendamment de celui de ses frères; dans l'héritage égalitaire sélectif les fils revendiquaient des droits égaux sur les biens ancestraux du père et sur les biens que la mère avait reçus en dot. Avec l'héritage, donc, les fils, et non les filles, prenaient possession des biens qui leur permettaient de fonder de nouveaux groupes familiaux.

14. La formule de la succession indivise agnatique et de l'héritage suspendu impose aux enfants de ne pas diviser le patrimoine et de demeurer ensemble dans la maison ancestrale comme une unique entité sociologique.

15. Groupe familial constitué de plusieurs frères, mariés et célibataires, résidant ensemble.

16. Le calcul de l'héritage légitime, la legittima, est complexe. Pour donner un exemple, si un père avait trois fils et deux filles, le patrimoine, au moment de la dévolution, était partagé en deux moitiés : une première moitié allait exclusivement aux agnats et était partagée en trois, c'est-à-dire par le nombre des héritiers de sexe masculin; une seconde moitié était partagée en cinq, c'est-à-dire par le total des héritiers de sexe masculin et de sexe féminin. La legittima de la femme correspondait donc à la valeur en argent de la cinquième partie à laquelle elle avait droit. Avec le temps, le calcul de la legittima est devenu de plus en plus favorable aux filles, jusqu'à la division égalitaire d'aujourd'hui. 
Les Dell'Angelo et les Marchi ont été l'objet d'une étude plus approfondie car ils représentent dans le réseau parental, de façon très visible, l'application d'une pratique matrimoniale que l'on rencontre sous des formes moins évidentes dans les autres familles de petits propriétaires : le modèle du renchaînement. Plus simplement, et sans vouloir limiter à cette seule définition la complexité d'une telle pratique, il y a renchaînement quand le mariage entre deux individus non consanguins est suivi d'un mariage entre deux consanguins des mariés ${ }^{17}$. À l'origine des mariages multiples entre deux familles, il y a la notion de proximité - sociale et spatiale - qui émerge très nettement des récits relatant les multiples mariages entre les Dell'Angelo et les Marchi :

- B. : «Nous, Dell'Angelo, on fréquentait Italo Marchi, parce qu’on était parents, par ma mère : la femme d'Italo et moi, on était cousins germains. On fréquentait beaucoup aussi Settimio, Domenico, on faisait tout ensemble : si l'un faisait quelque chose, les autres faisaient pareil. Imaginez, au lieu de quatre ou cinq maisons, là, à la Gorgone, c'était comme s'il y avait eu une cour, pas une vraie cour, une cour imaginaire.»

"Je l'ai bien connue, Teresa Dell'Angelo, elle habitait dans cette maison. Quand elle s'est mariée, elle est allée cinquante mètres plus bas, car elle a épousé Domenico Marchi qui habitait là » (Interview de B. Dell'Angelo).

- O.: «Les Dell'Angelo et les Marchi habitaient un petit hameau. Ce groupe était composé de personnes qui étaient toutes propriétaires et cultivaient elles-mêmes leurs terres; et vu les difficultés de déplacements, des systèmes de communications, c'est dans ce groupe de "Marchi et compagnie" que fleurissait cette jeunesse qui s'est mariée à deux pas de chez elle, sans aller à la recherche d'autres personnes de l'extérieur. Dans ce groupe, leurs enfants ont trouvé une jeunesse qui s'est à nouveau mariée [la $2^{e}$ génération], parce que les garçons et les filles étaient du même coin, ils avaient les mêmes habitudes et la même mentalité, finalement ça veut dire qu'entre eux ils ont trouvé la tranquillité» (Interview de O. Dell'Angelo).

17. La notion de "renchaînement » est utilisée par plusieurs auteurs de façon différente. Sur ce sujet, dans la littérature anthropologique, on distingue le renchaînement d'alliance (mariage entre individus qui partagent en commun soit un consanguin éloigné, soit un allié) et le redoublement d'alliance (mariage de deux ou plus consanguins avec $n$ consanguins d'une autre famille). Plus généralement, le "renchaînement " symbolise le renouvellement d'un lien entre des personnes déjà reliées par relation d'affinité. Un approfondissement des renchaînements produit une subdivision entre : 1) le renchaînement qui représente un échange direct simultané par deux couples de consanguins (comme dans le modèle de mariage double, parallèle ou croisé) ; 2) le renchaînement différé, fondé sur la notion de " partage en commun ", c'est-à-dire que le mariage unit des individus qui partagent un lien d'alliance (un consanguin ou un affin en commun), comme dans le mariage FZHZD où les époux qui redoublent l'alliance ont les cousins en commun. En ce qui concerne les figures précédentes, le lexique change chez d'autres chercheurs. On distingue entre : 1) les renchaînements de premier ordre ou binaires, comprenant un seul lien d'affinité préalable - deux liens d'affinité en tout - entre les conjoints (par exemple, mariages avec la ZHZ, FZSWBD) ; 2) les renchainements de second ordre ou ternaires, comprenant deux liens d'affinité préalables - trois en tout - entre les conjoints (comme dans le mariage ZHZHZ). On distingue également entre : 1) les renchaînements fondés sur un rapport de symétrie (les liens entre les deux couples des conjoints passent uniquement par la consanguinité), où se retrouvent les échanges directs simultanés et différés, précédemment cités ; 2) les renchaînements fondés sur un rapport d'asymétrie : dans ce cas, le lien passe par des personnes extérieures. Selon le classement précédent, cette dernière typologie appartiendrait au groupe des renchaînements ternaires. 
Ces descriptions évoquent la notion de proximité spatiale et parentale puisqu' " on était voisins et parents". L'aspect le plus intéressant, cependant, réside dans le fait que ces familles, portant les deux patronymes, étaient d'une dimension démographique suffisante pour soutenir dans le temps la pratique du renchaînement : si l'on considère que, outre le grand nombre d'enfants, il fallait également tenir compte de la différence d'âge entre les époux, on comprend l'importance de la dimension démographique de cette communauté parentale et de voisinage.

Le premier mariage entre les deux familles, dont on a retrouvé la trace dans les archives de l'église, remonte à la fin du XVIII siècle, le dernier a été célébré en 1928 ; pendant toute cette période on compte douze mariages entre les deux noms. Le plus grand nombre d'unions a lieu entre le début du XXe siècle et 1922 (six mariages). Bien sûr, les mariages célébrés ne se limitaient pas à ce réseau matrimonial, mais concernaient d'autres familles de petits propriétaires, de meuniers et aussi de métayers, avec certaines desquelles se répétait, sur le mode mineur, la pratique du renchaînement (voir infra figure 2).

Un des plus étonnants témoignages oraux - qui souligne le caractère systématique mais aussi casuel de ces mariages selon la perception du narrateur - est le suivant :

- O. : "Mon père, un vieux coureur de jupons, dans le vrai sens du terme, qui a été huit ans militaire à la frontière entre la Suisse et l'Italie, là-haut, où il était [à] Varese, les aventures... Il revient, et il renifle [il fait celui qui renifle] l'odeur de Pia Marchi, et alors il se marie avec."

Question : "Dans quel sens l'odeur : c'est une métaphore?»

- O. : «Une métaphore dans le sens où lui a vécu une jeunesse insouciante, d'une fille à l'autre, et alors il tombe follement amoureux de ma mère, et il se marie en un clin d'œil. Un homme avec de l'expérience qui, à un certain moment, revient et tombe dans les filets d'une Marchi. Cela veut dire que, ou bien la Marchi avait des grâces particulières, ou bien que..." (Interview de O. Dell'Angelo).

\section{Une famille de grands propriétaires : les Del Bravo}

Laissant de côté les raisons et les causes des renchaînements de l'alliance - qu'il serait simpliste de justifier exclusivement par la présence d'un patrimoine -, il est opportun maintenant d'examiner une autre famille importante de propriétaires terriens, pour apprécier ensuite, avec la théorie des graphes, les différences entre les réseaux matrimoniaux.

Le nom Del Bravo est porté par de nombreuses familles d'origines sociales bien différentes. Le groupe examiné dérive de Francesco Del Bravo, qui a vécu à la fin du XVII siècle : ses descendants sont mentionnés comme les grands propriétaires terriens de La Chiesina. Ils étaient proprié- 
taires d'une fattoria, c'est-à-dire une exploitation agricole composée de plusieurs métairies.

Question: «Pouvez-vous me raconter l'histoire de votre famille? Ce que vous avez entendu raconter? D'où ils venaient? »

- E. : "Moi, j'ai toujours su qu’ils ont vécu ici à La Chiesina. Mon père était "le patron Dino", mon grand-père était "le patron Checco", ma grand-mère "la patronne Marietta". Il n'y a pas grand-chose à raconter. Mon père était surtout un passionné de chevaux, de voitures... et de chasse!»(Interview de E. Del Bravo).

Les Del Bravo suivaient les règles de dévolution du patrimoine déjà rencontrées pour les Dell'Angelo et les Marchi (modèle de la succession segmentaire agnatique et de l'héritage égalitaire sélectif). Le développement économique de la famille atteint son apogée dans les années 1920 : dans les livres de comptes des métairies de la propriété, on lit que de 1925 à 1930, il y avait douze métairies, dont neuf de près de sept hectares et trois de près de dix hectares. Plus tard commença le déclin économique, jusqu'à la perte de presque tout le patrimoine foncier : Dino, futur chef de famille, dilapida le patrimoine familial tout entier à cause d'entreprises désastreuses, ce qui provoqua la «fin » de la famille et la mort soudaine de celui-ci en 1931. Avant la faillite financière, beaucoup de familles de métayers de La Chiesina gravitaient autour de cette famille:

- E. : "Il y avait une boutique. Ils [les paysans] allaient là, ils s'asseyaient devant, grand-père prenait un banc, avec le journal, ils écoutaient Don Checco: "patron Checco va nous lire le journal" et grand-père y allait et il leur lisait le journal. Après ils faisaient des commentaires, ce qu'ils avaient entendu dire, par-ci, par-là, suivant celui qui lisait. »

"Je ne me souviens plus comment il s'appelait, il était pauvre, si pauvre, c'était un pauvre homme, et je me souviens que moi j'étais petite. [...] Il venait chez nous : "Patronne, vous auriez pas une veste?" Et ma grand-mère répondait: "Oui, oui, je vous en donnerai une, la prochaine fois que vous viendrez ici”. Car il était pauvre, il venait d'une petite métairie : "ils n'y gagnaient pas un sou" - disait ma grand-mère "que veux-tu qu'ils y gagnent..." Une de ses filles était venue travailler comme bonne chez nous, une autre avait trouvé du travail comme bonne chez une tante qui habitait à Rome. [...] Quand les femmes venaient chez nous à Noël, elles nous apportaient des poulets et ma grand-mère, en échange, leur donnait à manger: "Venez, venez", puis elle leur préparait un tablier et un fichu à emporter, vous comprenez? On ne disait pas: "Moi, je suis le patron, toi tu es le paysan" (Interview de E. Del Bravo).

La famille Del Bravo, de par son statut social, se situait entre les petits propriétaires, qui avaient un niveau de vie bien plus bas, et les familles des grands propriétaires d'origine noble. L'analyse du réseau parental des Del Bravo, si on l'observe du point de vue de l'affinité, présente une structure "stellaire", "dispersée ", prédisposée à nouer des liens avec des familles lointaines et toujours différentes. Il n’y a pas de renchaînements, de 
mariages entre consanguins ou bien entre les affins des affins; les alliances se dispersent sur le territoire et arrivent à comprendre des familles résidant même très loin, au-dehors du cadre régional. Dans certains cas, il s'agit de mariages entre propriétaires, dans d'autres de mariages faisant suite à des connaissances occasionnelles ou des mariages organisés par les familles pour empêcher des mésalliances.

\section{Les familles de métayers}

À l'intérieur de la même aire territoriale, La Chiesina, il y avait les familles des métayers. À la différence des familles de petits propriétaires, comme les Dell'Angelo et les Marchi, les métayers ne possédaient pas la terre qu'ils travaillaient ${ }^{18}$, ce qui ne signifiait pas forcément une plus grande pauvreté. Mais c'est justement sur le concept de propriété familiale que l'on remarque la distinction entre métayers et petits propriétaires, surtout en ce qui concerne la valeur matrimoniale:

Question : «Et quels rapports y avait-il avec les familles de petits propriétaires?»

— F. : «Et bien, on se regardait un peu... Parce que, eux, ils pensaient qu'ils étaient supérieurs à nous, les paysans... Ce n’est pas qu'ils vivaient beaucoup mieux que nous ! Ils étaient comme nous! S'ils avaient dix, ils voulaient faire vingt, mais bon... ils pensaient qu'ils étaient mieux que nous, les femmes aussi. Les filles, bon, avant de prendre un paysan, de se marier avec un paysan, elles y regardaient» (Interview de F. Biagini).

Les Mancini et les Farnesi faisaient partie des familles de métayers les plus anciennes de La Chiesina et ce n'est pas un hasard si ce sont les patronymes prédominants d'un point de vue démographique dans la commune de Lucignano: le caractère sédentaire de ces familles de métayers (par rapport à l'instabilité résidentielle caractéristique de cette classe professionnelle), ainsi qu'un taux élevé de fécondité ont contribué à la diffusion de ces noms de famille sur le territoire.

On retrouve les Mancini dans l'État des âmes de 1890 ; le groupe domestique recensé est composé de treize personnes: Angelo est veuf, il réside avec les noyaux familiaux de trois de ses fils et avec un quatrième, célibataire; Luigi, frère d'Angelo, réside avec lui, ainsi que la famille qu'il a procréée. Les Farnesi sont réellement une «vieille " famille de La Chiesina :

18. La famille polynucléaire ou indivise des métayers était un groupe résidentiel composé de plusieurs familles nucléaires de générations différentes, apparentées selon l'axe de la cohésion consanguine paternelle. La résidence était patrivirilocale : après les noces, le nouveau couple vivait avec les parents et les frères de l'époux avec leurs familles. Les dimensions du groupe domestique étaient proportionnelles à la grandeur de la métairie (podere) et dans la phase de maximum démographique du cycle de développement domestique, la famille pouvait compter vingt ou même trente membres. Ensuite se produisait la scission d'un ou plusieurs noyaux pour reporter le groupe aux dimensions optimales; les noyaux qui s'étaient séparés allaient occuper des métairies libres de la même propriété (fattoria) ou d'autres propriétés. 
la souche des actuels descendants est Francesco, dans la seconde moitié du XVIII siècle. Au XIX siècle, les Farnesi étaient classés par le curé comme de "très pauvres travailleurs agricoles ", "travaillant pour autrui mais très nécessiteux ", et ils allaient le rester jusqu'en 1973, année où ils achetèrent le domaine agricole. Le groupe domestique multiple et patrivirilocal des familles de métayers est résumé, encore une fois, dans une remarque du curé, qui, à l'occasion de la mort du petit Angiolo en 1818, notait: «il était d'une famille très pauvre de travailleurs agricoles et lui survivent sa mère, un frère, des oncles paternels et des cousins ". Après quoi, il est intéressant d'observer la mémoire des deux familles:

Question: «Pouvez-vous me raconter exactement l'histoire des Mancini et des Farnesi ? Où habitaient-ils? D'où viennent-ils, comme famille? Que vous a-t-on raconté?»

- F. : «Mes parents ont toujours habité ici, à La Chiesina.»

- U. : «Non, nous, on a toujours habité ici, on n’a jamais bougé d'ici» (Interview de U. Mancini et D. Farnesi).

Les familles Mancini et Farnesi se fréquentaient depuis longtemps, ce qui produit une simplification du rituel des fiançailles (F. et U. se sont mariés en 1957), aspect qui apparaît également dans d'autres témoignages de fiançailles entre voisins :

- R. : «Nous, les familles, on se connaissait... on se connaissait depuis trop longtemps, donc ça a été... il n'y a pas eu besoin de faire les "escaliers" ${ }^{19}$. Il y avait la formule pour demander la main. Eux, au contraire, ils se sont demandé la main entre eux et c'est tout" (Interview de U. Mancini, R. Mancini et D. Farnesi).

\section{Le réseau matrimonial "non patrimonial” des métayers}

On a observé précédemment que les noms des petits propriétaires de La Chiesina sont inscrits dans le réseau matrimonial du hameau de Gorgone, dont la structure portante est fondée sur les renchaînements entre les Dell'Angelo et les Marchi; un second réseau matrimonial «non patrimonial » - qui appuie sa physionomie sociale et économique sur l'absence d'un véritable patrimoine familial - comprend principalement les familles de métayers de La Chiesina. Ce second réseau matrimonial montre certainement de nombreux échanges multiples (renchaînements binaires) entre familles, mais pas avec la même continuité et la même fréquence que celles

19. Au début du $\mathrm{XX}^{\mathrm{e}}$ siècle, l'usage voulait que la belle-fille, au moment où elle entrait dans la maison de son mari après le mariage, montât les marches jusqu'à la moitié, là où sa belle-mère l'attendait, et lui demandât: "Maman, voulez-vous de moi ?" À cette question, la belle-mère répondait : "Mais oui, ma chère!» Après quoi les deux femmes s'embrassaient. Dans les années 1950 , cet usage avait pratiquement disparu. 
visibles dans le réseau des propriétaires de Gorgone. En d'autres termes, les renchaînements entre métayers peuvent concerner trois générations au maximum; ils ne s'étendent certainement pas sur trois siècles, du XVIII ${ }^{\mathrm{e}}$ au XXe siècle, comme pour les Dell'Angelo et les Marchi. De plus, la mobilité territoriale des métayers, plus grande que celle des propriétaires, aurait de toute façon empêché les métayers d'appliquer le renchaînement de l'alliance tout au long des générations. À la fin, peut-être les renchaînements n'étaientils pas « nécessaires » aux métayers, ce qui renforce l'hypothèse qu'il s'agissait de stratégies matrimoniales appliquées pour défendre les patrimoines, les petits patrimoines. Le sens de cette stratégie d'alliance, toutefois, ne se limitait pas aux questions patrimoniales : la valeur qui caractérisait la candidature du propriétaire était aussi un trait de l'identité, de sa mémoire familiale.

L'analyse du réseau matrimonial des familles des métayers de La Chiesina relève des « circuits » plus que des mariages multiples, c'est-à-dire des chaînes matrimoniales de différentes longueurs qui, tôt ou tard, reviennent au point de départ (cf. figure 3). Ce n'est pas tout : tandis que le réseau des propriétaires donne de la valeur aux alliés qui, à la longue, deviennent des consanguins, le réseau des métayers semble valoriser les affins des affins. Et puisque le réseau n'est autre, selon moi, que la représentation généalogique de bassins matrimoniaux spécifiques, il est possible de conclure que ces derniers sont à la fois une entité spatiale (Gorgone, La Chiesina,...) et un système d'affinités qui se modèle sur les caractéristiques sociales de ses composantes. Cela sera plus clairement visible si on le soumet à un agrandissement des portions de réseaux parentaux décrits ci-dessus par la théorie des graphes.

\section{Les configurations stellaires, centripètes et les circuits}

Les actes de mariage apportent jusqu'en 1973 des renseignements concernant les parents des époux, donc sur le mariage contracté par les ascendants directs des époux; il est possible, à partir de ces informations, de construire des parentèles, des réseaux multicentrés, des agrégats de noms de famille. L'analyse des réseaux sociaux (social network analysis) a permis d'élaborer des programmes ${ }^{20}$ afin d'explorer et de définir les caractéristiques des réseaux; elle fonde sa théorie sur la notion de structure sociale comme réseau de relations sociales. L’importance des réseaux est due au fait qu'à l'intérieur de ceux-ci sont transmis des modèles comportementaux, des informations, des biens.

20. On fait référence au programme Pajek 0.79, réalisé par Vladimir Batagelj et Andrej Mrvar (De Nooy, Mrvar \& Batagelj 2004). Avec Pajek, j'ai utilisé Expliens V.2f, application sur Access pour l'étude des données relationnelles, élaborée par Pascal Cristofoli pour l'Atelier des données relationnelles Ehess (Laboratoire de démographie historique) - Ined (Histoire critique des sources et des méthodes). 


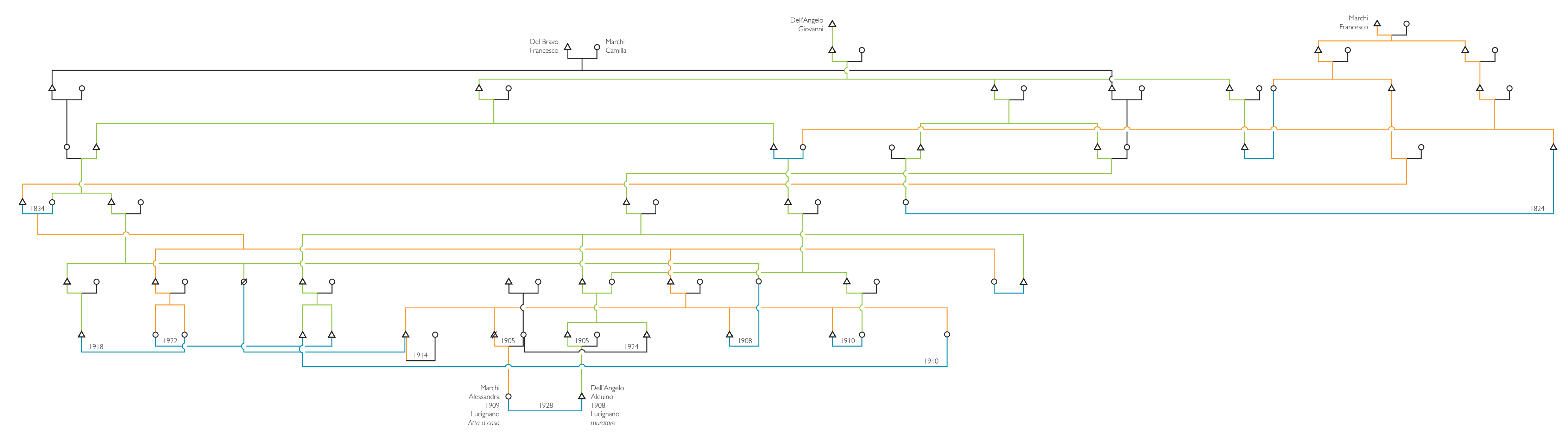

- Réseau matrimonial des Dell'Angel

- Réseau matrimonial des Marchi 


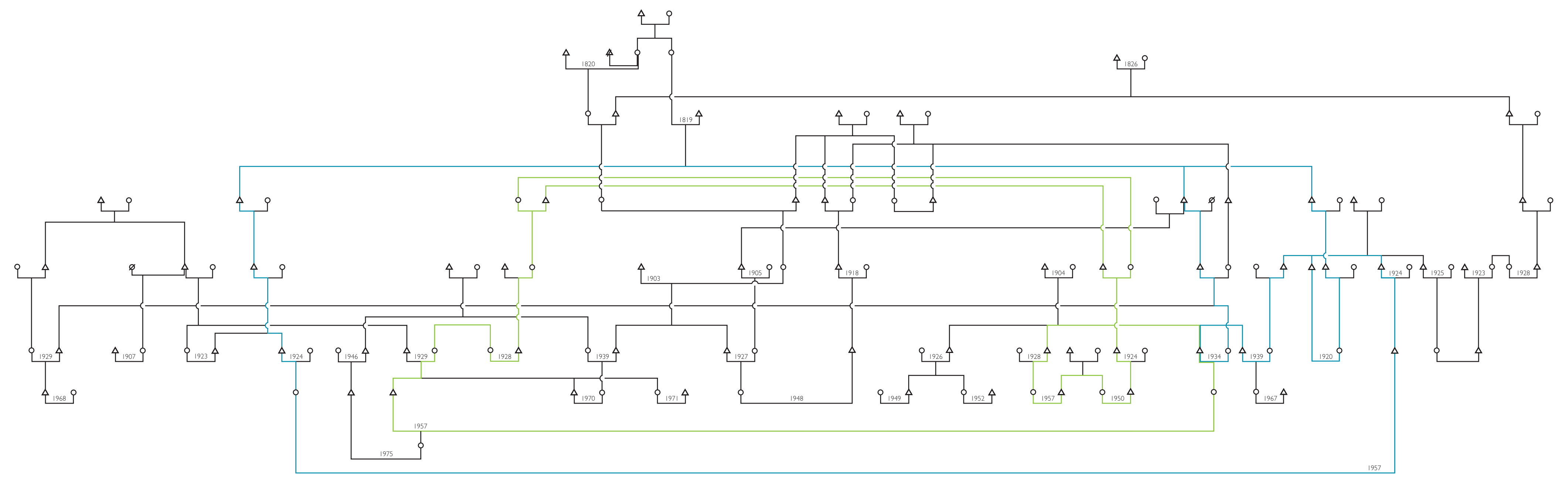

E E Exemples de circuit 
L'analyse des réseaux sociaux produit donc des études statistiques et des mesures de systèmes multirelationnels; elle se distingue des méthodes traditionnelles par l'inclusion de concepts et d'informations sur les relations entre acteurs et par le relevé des propriétés du système relationnel. La représentation de l'ensemble des liens dans un espace bidimensionnel est appelée "graphe»: un graphe est composé d'une série de points - qui représentent des individus - et d'une série de lignes reliant les points entre eux - qui représentent les relations. Dans ce paragraphe, le réseau matrimonial sera observé dans sa totalité ou configuration (Houseman \& White 1996) ${ }^{21}$.

Un acte de mariage contient trois mariages - celui des mariés, celui des parents du marié, celui des parents de la mariée - et quatre liens de filiation : deux de maternité (les mariés et la mère respective) et deux de paternité (les mariés et le père respectif). La traduction graphique (d'après le programme Pajek) des informations contenues dans un acte de mariage est la suivante:

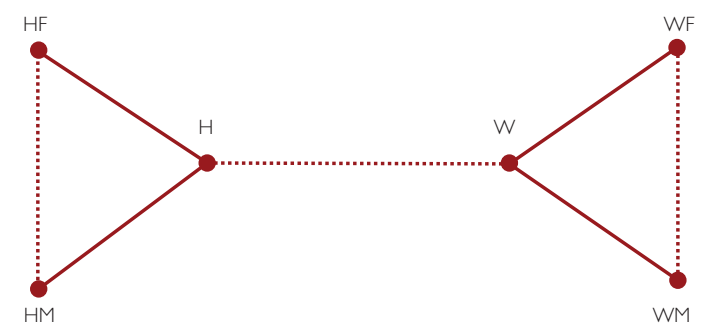

Fig. 4 Représentation graphique des relations contenues dans un acte de mariage

Les lignes en pointillés indiquent les mariages: la ligne centrale représente le mariage des époux nommés dans l'acte, tandis que les deux lignes aux extrémités représentent les mariages des parents des mariés. Les lignes diagonales continues correspondent aux liens de filiation: liens de paternité et de maternité entre chacun des mariés et ses parents respectifs.

La représentation est un graphe composé de sommets (les individus présents dans l'acte du mariage) et de lignes entre paires de sommets : les lignes symbolisent les relations de mariage (lignes en pointillés) et de consanguinité (ligne continue). La disposition des points sur le plan

21. Pour les deux auteurs, l'interprétation du réseau dans sa totalité repose sur l'idée suivante: chaque mariage est en partie conditionné par des mariages qui le précèdent, et contribue, à son tour, à la détermination des unions suivantes. 
répond au principe de "proximité relationnelle» ou de "synergie algorithmique ${ }^{22}$ : le concept de fond est que la présence d'un lien se traduit en une force d'attraction qui rapproche les points (individus) concernés, tandis que l'absence de relations correspond à une force de répulsion qui les éloigne. Le graphe final représente le point d'équilibre dans la disposition des individus et des liens entre eux (Fruchterman Reingold algorithm). La traduction graphique des relations contenues dans un acte de mariage constitue le module de base dans la construction des réseaux matrimoniaux; à partir de cette forme élémentaire d'autres développements graphiques sont possibles.

Il est important de souligner que les graphes figurant dans le texte sont formés de relations multiples, c'est-à-dire de la combinaison des rapports de consanguinité et de mariage ; pour chaque cas, on a effectué une sélection des liens afin d'accentuer les caractéristiques morphologiques du graphe $^{23}$. Une autre propriété de ces réseaux est qu'ils sont "sociocentrés " et non «egocentrés ", c'est-à-dire qu'il n'y a pas d'acteur focal. Les relations entre paire d'acteurs sont non directionnelles (nondirectional relation $)^{24}$ et dichotomiques (dichotomous relation/nonvalued relation) ${ }^{25}$. Dans cet essai, l'évaluation de la fréquence, de la force et de l'intensité des liens, et l'analyse du système relationnel d'un potentiel Ego ne constituent pas l'objectif prioritaire; l'objet de la réflexion est, en revanche, la morphologie du réseau matrimonial dans sa totalité.

22. Pajek calcule la distance entre les points de connexion et celle entre les points de nonconnexion : le résultat fournit la disposition des éléments et donc la représentation du graphe.

23. La construction des graphes à relations multiples a été réalisée avec Expliens (cf. supra note 20). Le programme construit le dictionnaire des Ego (les acteurs) et celui des liens (la liste de toutes les relations entre les acteurs). Chaque acteur est représenté par un numéro et constitue un sommet du graphe; les relations entre acteurs sont représentées par les numéros des sommets correspondants et sont indexées sur la base de la nature du lien (paternité/maternité, mariage).

24. Les relations du mariage et de consanguinité ne sont pas représentées comme directionnelles (directional relation) parce que l'affirmation selon laquelle " $\mathrm{n}$ est marié avec $\mathrm{n} 1$ » ou bien « $\mathrm{n}$ est consanguin de $\mathrm{n} 1$ " équivaut à l'affirmation " $\mathrm{n} 1$ est marié à $\mathrm{n}$ " ou " $\mathrm{n} 1$ est consanguin de $\mathrm{n}$ ". Dans la réception d'un message à l'intérieur d'un groupe d'acteurs, en revanche, les lignes sont directionnelles parce que l'affirmation " $\mathrm{n}$ envoie le message à $\mathrm{n} 1$ " n'est pas équivalente à l'affirmation " $\mathrm{n} 1$ reçoit le message de $\mathrm{n}$ ": dans ce cas la ligne entre les deux sommets a une direction de $\mathrm{n}$ à $\mathrm{n} 1$ et est représentée par une flèche (Wasserman \& Faust 1994).

25. Dans la construction des graphes, une relation est dichotomique quand le lien existant entre deux acteurs - par exemple la relation de consanguinité ou de mariage - est chiffré comme absent (0) ou présent (1). Au contraire, par le terme anglais valued relation sont représentées la force, l'intensité et la fréquence d'un lien entre acteurs. En présence d'un groupe Ego-focus (groupes qui ont Ego comme centre) ou ancestor-focus (point de vue de l'ancêtre pour la formation des groupes), les deux premières qualités - la force et l'intensité du lien - pourraient signifier la distance généalogique entre Ego et les Alters ou entre l'ancêtre et ses descendants. 
Ainsi, de l'analyse des données généalogiques et des mémoires de famille, on déduit que le réseau parental des grands propriétaires a une structure «stellaire» ou "radiale» (graphe stellaire) et le réseau des petits et moyens propriétaires a une structure "centripète», tandis que celle des métayers peut se positionner à un niveau intermédiaire, donnant lieu à une sorte de "circuit long". Le "graphe stellaire» représente un réseau parental où, à chaque génération, des noms de famille sont capturés; le modèle stellaire - une sorte de "paradigme" - exige que les patronymes inclus dans le réseau parental n'aient entre eux aucun lien de parenté (ni consanguins ni alliés à la suite des précédents mariages) : la structure, donc, se nourrit de nombreux patronymes. Le graphe suivant constitue le modèle de référence :

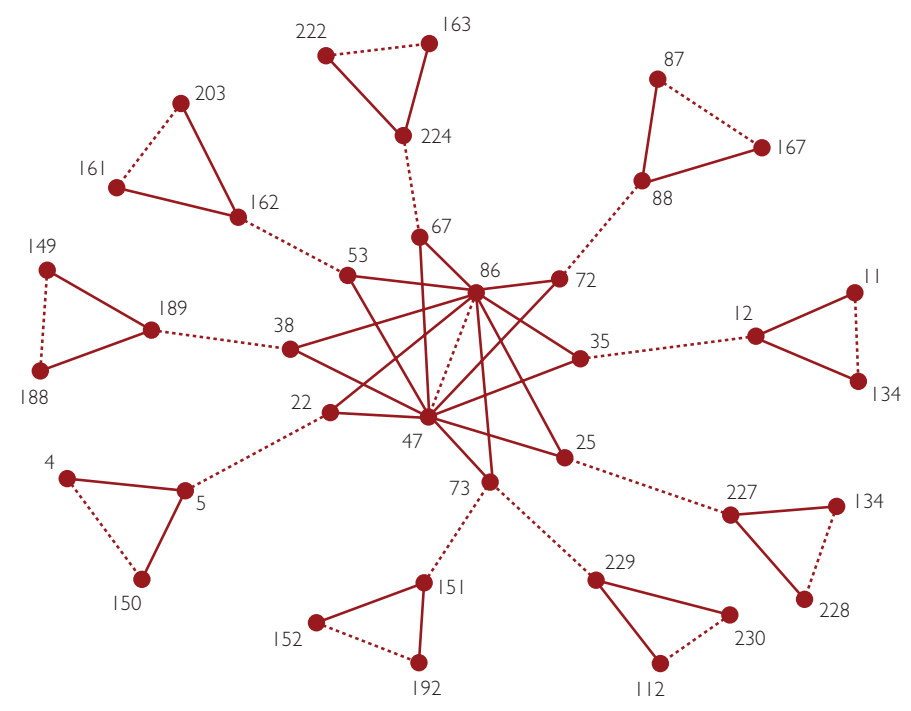

Fig. 5 Modèle de graphe stellaire (d'après le programme Pajek) ${ }^{26}$

Un cas concret reproduisant le modèle proposé ci-dessus est représenté par le réseau matrimonial des Del Bravo, les grands propriétaires de La Chiesina qui ont été décrits précédemment. La traduction en graphe des liens de consanguinité et surtout d'alliance des Del Bravo est la suivante:

26. Dans cette représentation, on distingue un couple d'origine ( ${ }^{\circ} 86$ et 47 ) qui a eu huit enfants $\left(\mathrm{n}^{\circ} 67,53,38,22,73,25,35,72\right)$; chaque enfant est marié (67 avec 224; 53 avec 162; 73 avec 151 et, en secondes noces, avec 229 , etc.) et le graphe indique aussi les liens de paternité et de maternité de chaque conjoint (222 et 163 sont les parents de 224;150 et 4 sont les parents de 5, etc.). 


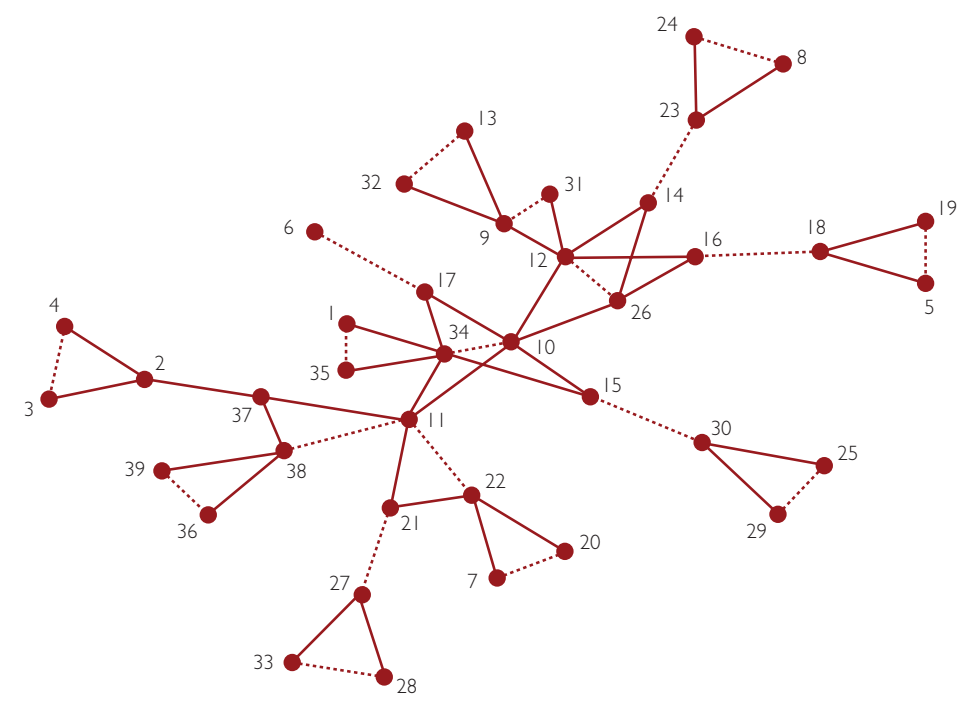

Fig. 6 Graphe du réseau parental (détail) des Del Bravo (d'après Pajek) ${ }^{27}$

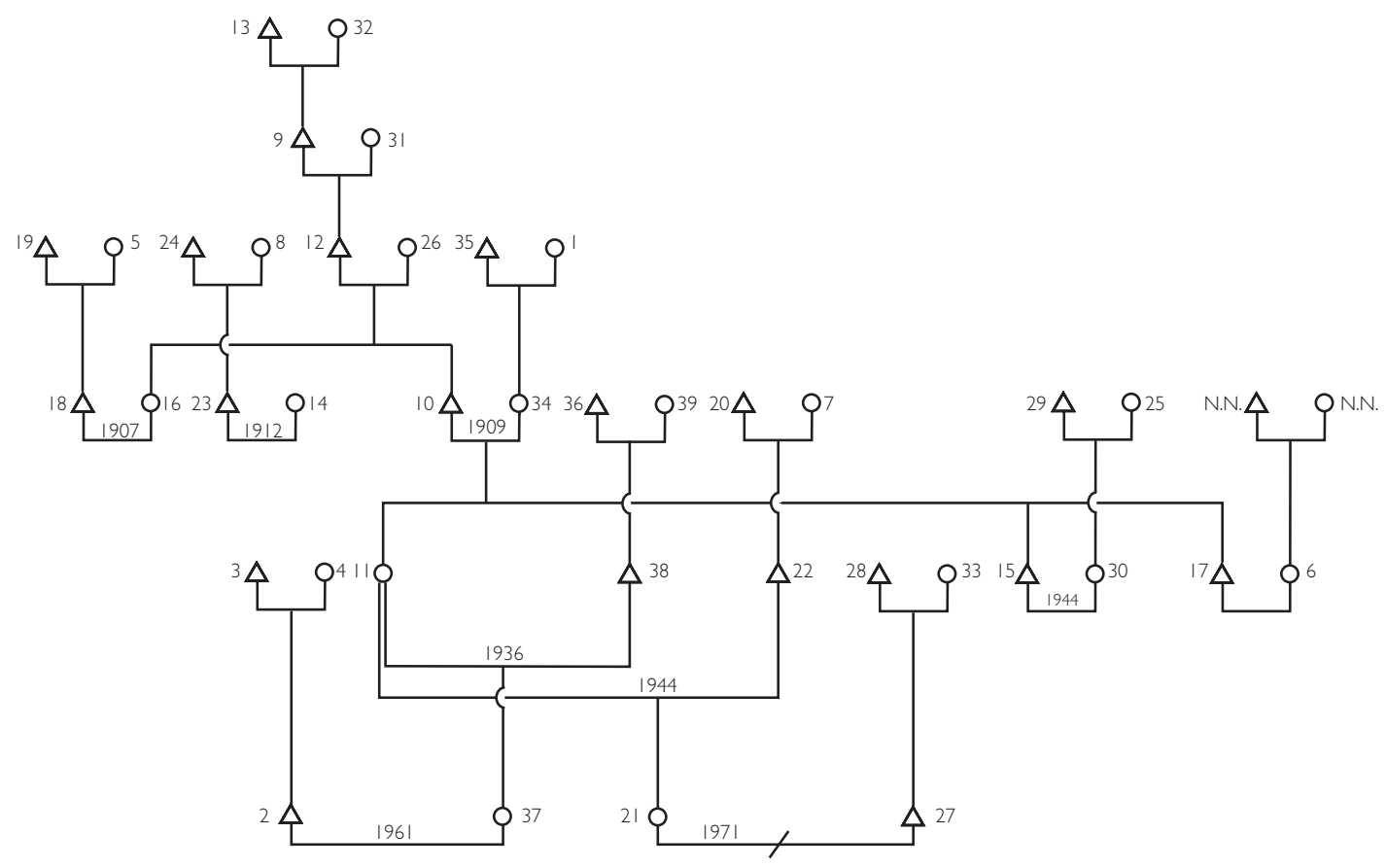

Fig. 7 Traduction en termes généalogiques de la figure 6

27. Chaque numéro correspond à un individu du réseau parental. Il n'est pas possible de reporter ici l'entière généalogie, où le modèle stellaire est plus visible. 
Le "graphe centripète» est un réseau multicentré sur des noyaux consanguins, alliés entre eux, qui se répètent tout au long des générations : c'est le cas des renchaînements entre les Dell'Angelo et les Marchi, où sont reproduits les anciens liens d'alliance, avec pour conséquence la répétition des patronymes au cours du temps. Le modèle ci-dessous représente un détail du réseau matrimonial général des deux familles :

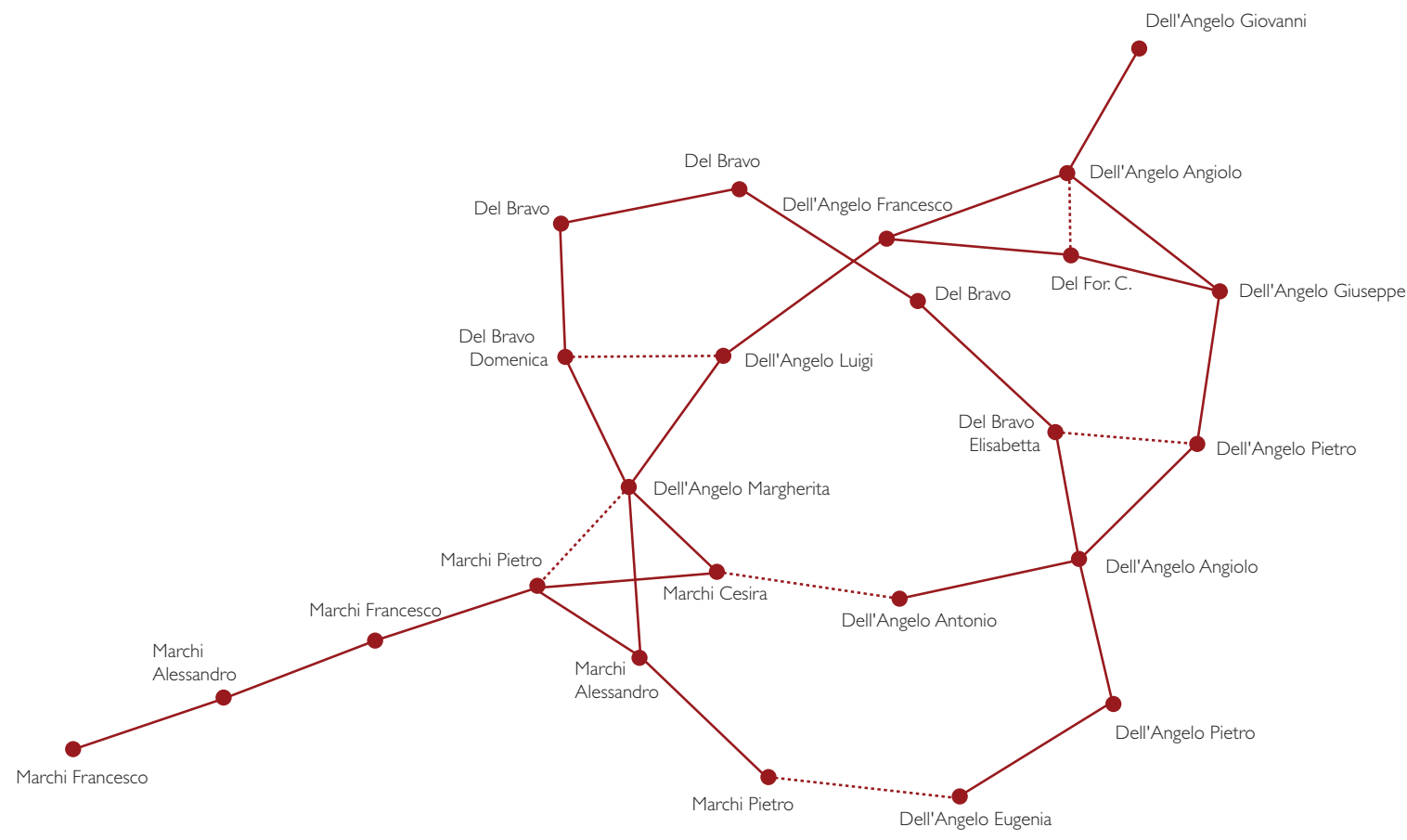

Fig. 8 Modèle à structure centripète.

Graphe du réseau parental (détail) des Dell'Angelo et des Marchi (d'après le programme Pajek)

Ce type d'alliance avait été décrit dans le paragraphe précédent : dans ce détail, les renchaînements initiaux ont déjà produit, au fil des générations, des mariages consanguins, tout en conservant la physionomie du renchâ̂nement clairement visible dans le mariage de Pietro avec la " cousine de sa cousine ", Eugenia (FZHBD).

La traduction graphique de la généalogie du programme Pajek montre un graphe qui revient continuellement sur lui-même, se noue, en créant de nombreux et brefs circuits à l'intérieur de la configuration générale; les deux extrémités latérales au noyau central sont les ancêtres des deux familles : cela rend évident le point de départ des deux alliances, à savoir 
le modèle du renchaînement et non le modèle du mariage à l'intérieur de la consanguinité. Dans ce deuxième cas, en effet, la représentation n'aurait montré qu'une seule extrémité; en outre, les anneaux centraux rendent visible le phénomène qui « consanguinise » deux parentèles alliées (HéritierAugé \& Copet-Rougier 1991).

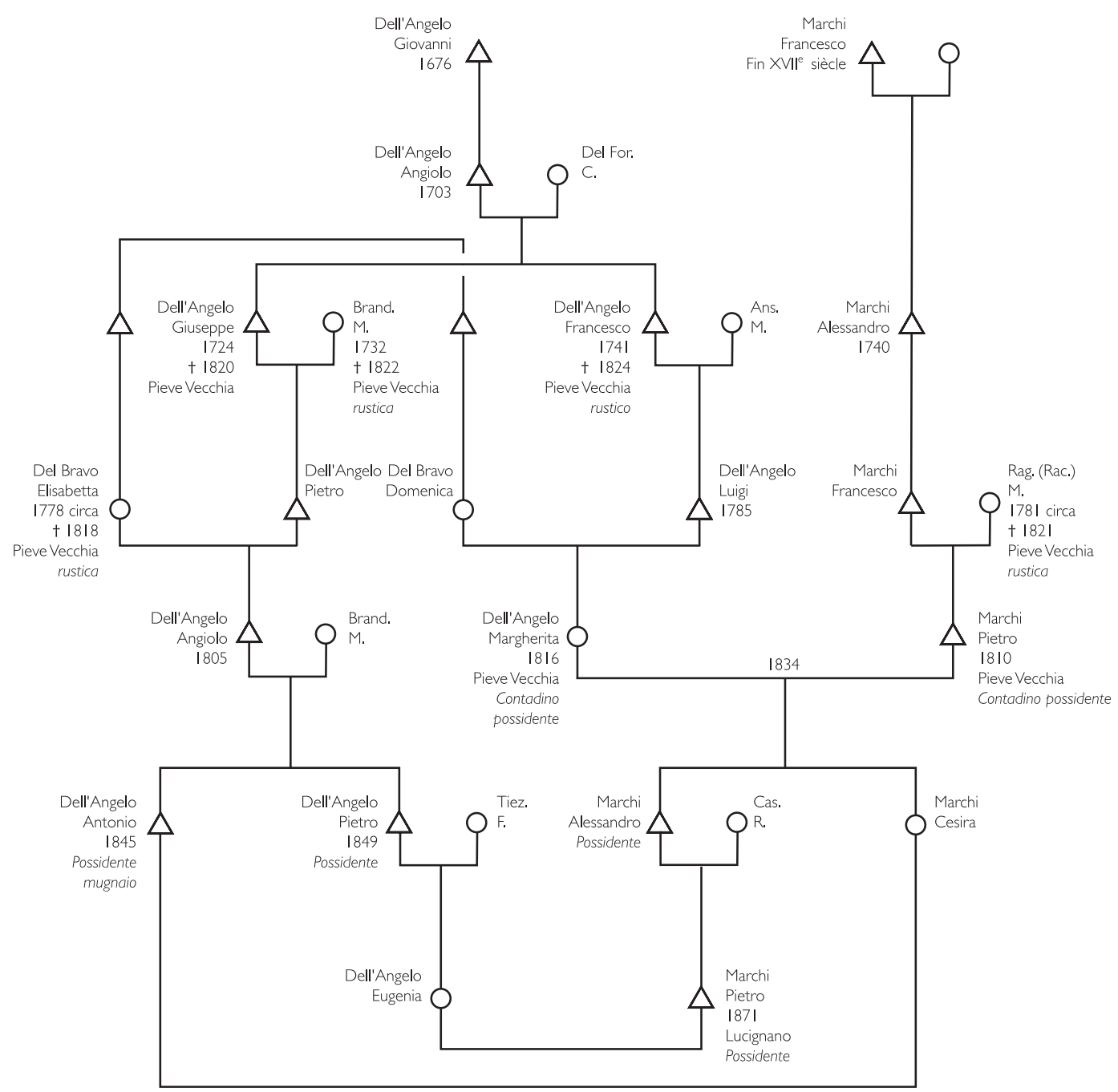

Fig. 9 Traduction en termes généalogiques de la figure 8 
Le "circuit long» - en référence à la structure du réseau parental des métayers - représente un réseau multicentré sur des noyaux consanguins qui changent au fil du temps; toutefois, contrairement au graphe stellaire qui ne se referme pas, le circuit revient tôt ou tard sur lui-même. Cela est rendu possible par le fait que les nouvelles alliances se développent à partir des connexions produites par les affins, au lieu de renouveler les anciens liens d'alliances. En d'autres termes, les mariages des générations successives sont stipulés non avec les affins mais avec les affins des affins et avec les affins des affins des affins. Le modèle de référence, qui concerne les familles de métayers Mancini, Farnesi, Furli et Biagini, est le suivant:

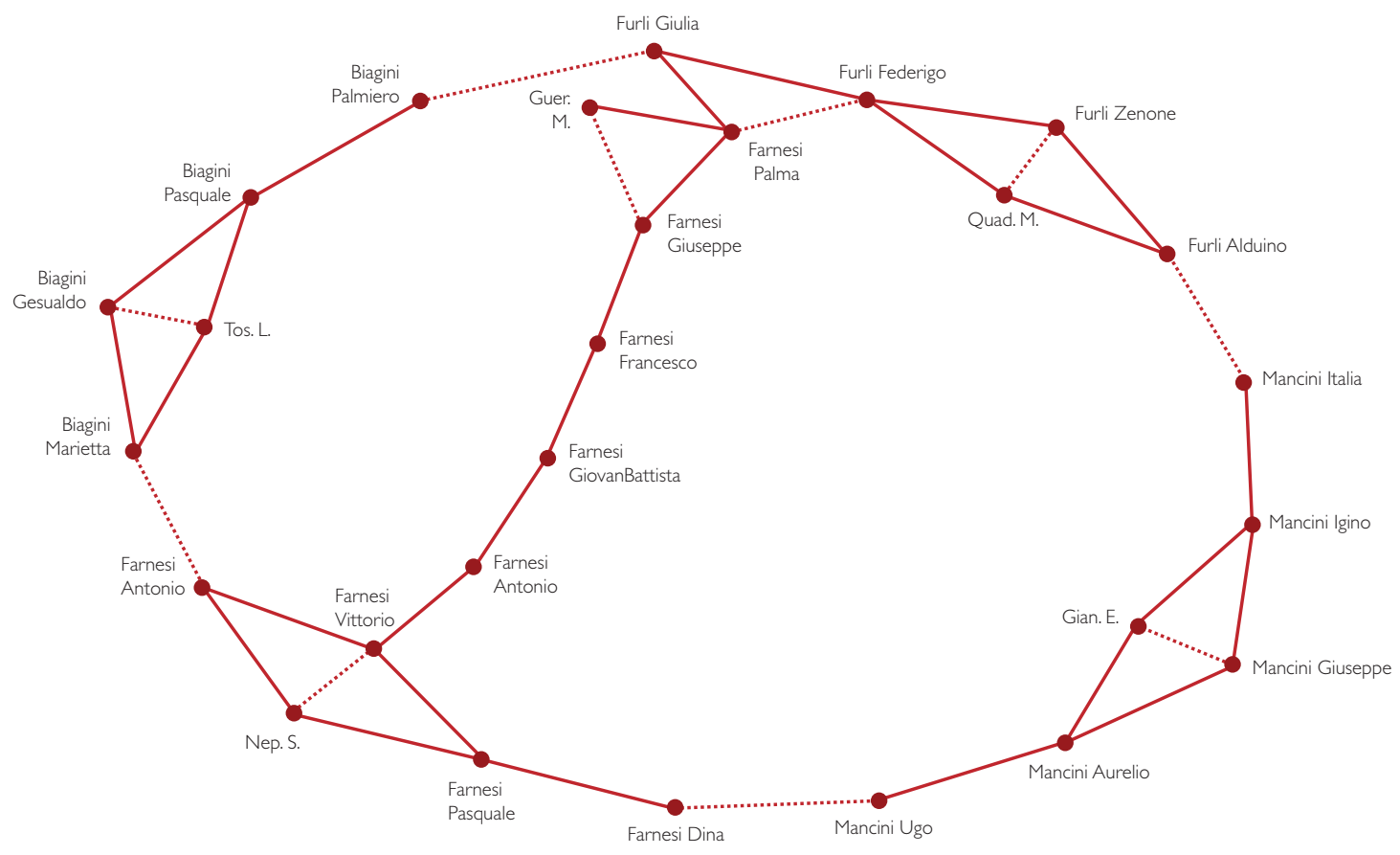

Fig. 10 Modèle de graphe en "circuit long".

Réseau parental (détail) des familles Mancini, Farnesi, Furli et Biagini

En bref, il est possible de construire un continuum dans lequel, à l'une des extrémités se trouve la configuration stellaire, à l'autre la structure centripète produite par l'application, à chaque génération, de renchaînements et de mariages dans la consanguinité, tandis que dans la position intermédiaire se trouve le circuit, c'est-à-dire une sorte de chaîne qui, à un certain moment, revient au point de départ. Il faut ajouter, cependant, que l'on peut observer des différences plus nettes entre la configuration stellaire et les deux autres configurations qu'entre la structure centripète et le circuit. 


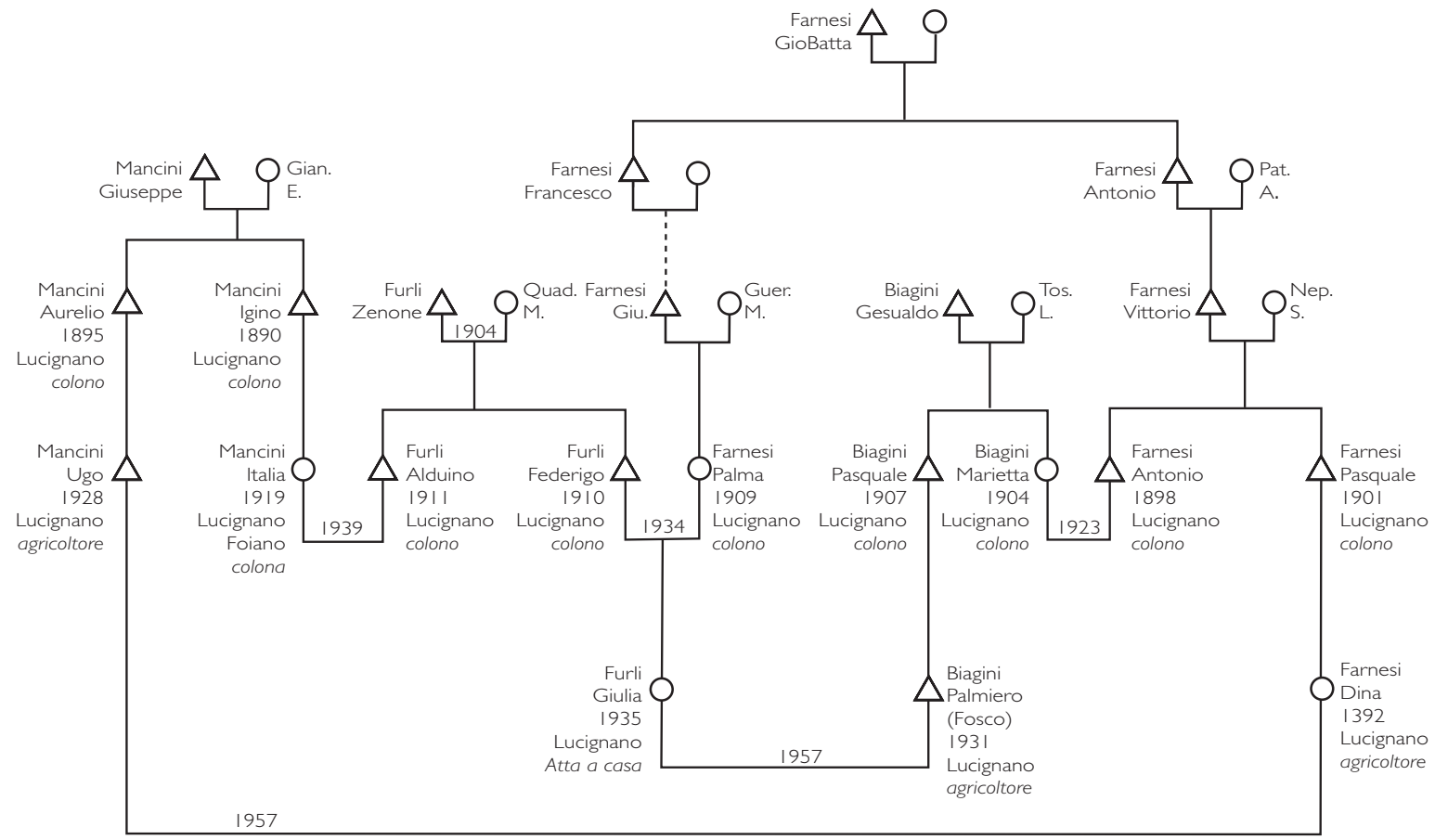

Fig. II Traduction, en termes généalogiques, de la figure 10

La différence entre ces deux dernières configurations peut être résumée et comprise selon la même distinction que celle effectuée par les anthropologues entre renchaînements binaires et ternaires, fondée sur la quantité de liens d'affinités préliminaires comptés entre l'époux et l'épouse concernés par le renchaînement de l'alliance ${ }^{28}$. C'est la "propension» du réseau à constituer des circuits plus ou moins longs - c'est-à-dire à valoriser les affins ou les affins des affins - qui produit une structure centripète ou un circuit long, tandis que la configuration stellaire se caractérise par l'attention à ne pas répéter les liens préliminaires d'affinité et de consanguinité.

L'hypothèse de fond consiste à considérer chacune de ces configurations de réseau caractéristique de certains groupes socioprofessionnels: les réseaux matrimoniaux de grands propriétaires, petits propriétaires et métayers tendent vers ces modèles. Somme toute, étant donné que ce qui les différencie est la notion de propriété, on peut se demander si ces réseaux parentaux ne cachent pas leur réelle physionomie, à savoir que ce 
sont des réseaux «patrimoniaux ». C'est-à-dire que le continuum entre une configuration stellaire et un circuit ne représente pas seulement une façon d'organiser les groupes des affins, mais fonctionne aussi comme indicateur de la valeur économique et symbolique du réseau.

\section{Le discours émique: la "famille", la "tribu", la "parenté"}

Les trois graphes proposés montrent - de manière plus nette que ne le fait la représentation généalogique - les caractéristiques des réseaux socioprofessionnels et leur propension variable à la cooptation des patronymes. Autrement dit, il y a des configurations de réseau programmées pour " consommer » des noms et des configurations de réseau prédisposées pour réduire au minimum les effets de la cooptation, qui finissent par se concentrer sur un nombre restreint de noms qui se répètent au fil des générations. Dans ce sens, la configuration stellaire est celle qui implique dans une plus ample mesure la dispersion du patronyme de référence à l'intérieur de nombreuses familles, qui diffuse le potentiel démographique dans des groupes parentaux toujours différents à chaque génération; la configuration centripète, à l'inverse, dessine une aire de choix du conjoint composée de peu de patronymes qui suffisent à la demande d'un conjoint à travers la répétition des mêmes liens au cours du temps. Le circuit présente les caractéristiques des deux configurations précédemment définies : d'abord il est programmé pour se déplacer d'une famille à l'autre, ensuite pour revenir au point de départ et composer un réseau formellement clos.

Là où ces trois typologies de réseau se dénouent (configurations de bassins matrimoniaux spécifiques), le discours émique a des nuances de lexique qui méritent un approfondissement, tout d'abord pour des termes tels que "famille», "tribu» et "parenté " (parentiera). Dans les deux extraits d'interview qui vont suivre, la notion de famille provient de la configuration centripète, c'est-à-dire du hameau de la Gorgone, habité par les Marchi et par les Dell'Angelo:

Questions : "Quelles étaient les familles dont vous entendiez le plus parler et que vous fréquentiez? Avec lesquelles aviez-vous le plus de rapports?"

- B. : "On avait le plus de rapports avec les voisins, c'étaient à la fois des parents et des voisins : les Marchi. On faisait beaucoup de choses avec eux : chacun faisait ce que les autres faisaient. La Gorgone était composée de différentes habitations, mais c'était comme si on avait habité dans une cour, on faisait tout ensemble " (Interview de B. Dell'Angelo).

- O. : "Ils habitaient tous à la Gorgone, dans un hameau. Dans une maison, il y avait le maire [les ancêtres du maire de Lucignano, aujourd'hui], le "sourd" habitait à côté, Rose au milieu, et puis le groupe de Quintilio Marchi, tandis que Venturino habitait le long de la route. C'était tout un groupe! Toute une famille! Toute une famille!» (Interview de O. Dell'Angelo). 
Le mélange de voisinage, consanguinité et affinité produit la « famille », c'est-

à-dire la participation de plusieurs agrégats de noms Dell'Angelo et Marchi à la fondation d'une unique descendance, où les noms de famille restent distincts mais les lignes généalogiques se trouvent irrémédiablement enchevêtrées. Alliance et consanguinité s'appliquent l'une sur l'autre et produisent une famille de parents et de voisins, de consanguins et affins. Il est important de souligner que ces regroupements parentaux, bien qu'ils soient engagés au même titre dans un programme reproductif identique, restent distincts dans l'histoire familiale et les stratégies économiques et sociales.

La famille dont parle E. Del Bravo (configuration stellaire) est essentiellement une entreprise formée par le chef de l'exploitation agricole et par ses paysans, dans une représentation paternaliste et communautaire de La Chiesina, et elle ne ressort jamais en tant que mot dans la description du réseau matrimonial des Del Bravo:

- E. : «Ces paysans, ils venaient chez nous jouer aux cartes pendant que les femmes cousaient ou tricotaient. On était tous dans la cuisine. Parce qu’on était tous une famille : c'étaient pas des domestiques, ils étaient de la maison" (Interview de E. Del Bravo).

Il ressort de ce témoignage une sorte d'idéologie communautaire, un modèle particulier de communauté où l'on regrette le type de relations interpersonnelles d'autrefois ; une idéologie qui met l'accent sur le sentiment d'égalité intrinsèque à ce type idéal de communauté non hiérarchisée: «ce n'étaient pas des domestiques, ils étaient de la maison", «nous, on était une famille». L'évocation nostalgique du passé, après le déclin économique de la famille Del Bravo et la désagrégation du tissu social de La Chiesina avec la fin du métayage, n’arrive pas à masquer complètement la pauvreté et la structure hiérarchique de cette communauté, visibles dans certains extraits d'interview déjà rapportés. Cette notion de famille-communauté est ultérieurement approfondie par d'autres familles de La Chiesina et ressort de l'analyse du "circuit»:

Question: "Quels étaient vos amis, les familles avec lesquelles vous aviez le plus de rapports?"

— F. : "Les Mancini! Et puis les Santi, les Cardelli : à La Chiesina, dans les années 1950, on était tous une famille" (Interview de F. Biagini).

Question : "Ces familles, quand vous étiez petits, elles se fréquentaient?»

— U. : «Oui, toujours très bien, toujours. Avec Biagini, on s'estime comme des frères, parce qu'on travaille ensemble depuis des années, on fait tout ensemble, on s'estime. J'estime Giovanna Cardelli et sa mère, des fois, je la prends pour la mienne : le soir on se promène bras dessus et, après la messe, je la ramène à la maison. On s'estime comme... même plus que si on était de la même famille! Avec Biagini, c'est pareil. On s'estime tous ici. Là, je travaille avec Biagini et on a acheté du matériel agricole ensemble, et on fait les moissons ensemble» (Interview de U. Mancini). 
Le mot famille est utilisé dans ce témoignage pour souligner le sens de "familiarité " que l'on ressentait dans la communauté de La Chiesina ; c'est-à-dire que dans ce hameau, les rapports de voisinage et d'amitié étaient des rapports de famille. Malgré tout, il faut distinguer entre la " famille » des parents et voisins de la configuration centripète et la famille des voisins qui sont "comme des frères" du circuit, résumée dans cette très belle phrase: "des fois, je prends sa mère pour la mienne". Les rapports interpersonnels fondés sur le code de comportement du "comme si ", c'est-à-dire du "comme des frères ", "comme si on était de la même famille", ne produisent pas de consanguinité, mais de l'" estime».

L'expression "famille», toutefois, peut réellement être étendue à tout le tissu social de La Chiesina et comprise dans sa signification réelle et non métaphorique, justement parce que les liens d'affinité et de consanguinité couraient d'une famille à l'autre, finissant par constituer des circuits d'affinité d'extension variée. Les significations et les effets sociaux et économiques, même secondaires, produits par le circuit doivent être pris au sérieux, puisqu'ils sont à la source de cette forme de familiarité - l'" estime» - dont on parle dans les témoignages, comme expression symbolique d'une aire privilégiée de choix du conjoint : le bassin matrimonial où « on s'estime comme des frères ", "attention à ne pas toucher à quelqu'un de La Chiesina ».

En approfondissant ultérieurement l'analyse, en sachant qu'il s'agit d'une interprétation théorique forcée, que signifie s'estimer "comme... plus que la famille" ? Dans le discours émique, il émerge un autre terme pour définir les habitants de La Chiesina:

Question : "On me disait qu’à Lucignano, on vous appelait la "tribu" : qu'est-ce que cela veut dire?"

— U. : «Nous, on s'entendait tous, rien à dire là-dessus, et s'il y a quelque chose... [on s'aide]."

Question : "Et la "tribu”, qu'est-ce que ça veut dire?»

— U. : "Mon frère, on lui disait qu'il était le "maire", ici. "

— F. : «Si vous voulez voir, venez un soir, l'été, vous voyez, là, devant chez moi.»

- U. : «On est tous là, le soir, jusqu'à dix, onze heures, tant qu’on n’éteint pas la lumière, on est tous là.»

- F.: «Si vous saviez ce qu'on nous envie... !»

- U. : «Même les habitants du Pozzo [un hameau de Foiano, une commune limitrophe], on les appelle la tribu, eux aussi ils sont unis, Sainte Vierge! Et nous aussi : ici, s'il y en a un qui touche à l'un d'entre nous, soyez tranquille, on s'en occupe!"

Question : "Oui, mais si je peux vous le demander, parce que ça m’intéresse, ces rapports de solidarité... dans le hameau de la Gorgone, était-ce pareil ?"

— U. : «Un peu moins, c'est moi qui vous le dis...» 
Question : «Et pourquoi, d'après vous?"

— U. : "Qui sait ? L'envie. Ou bien chacun pense pour soi... il y en a...» (Interview de U. Mancini et D. Farnesi).

— E. : «La Chiesina ? On l'appelait la tribu : ils étaient unis, unis. »

Question : «Et vous avez d'autres exemples de cette solidarité entre les habitants de La Chiesina?"

— E. : "Ceux de La Chiesina, ils étaient comme ça : il fallait pas toucher à l'un d'entre eux! Si un gars de Lucignano touchait à un gars de La Chiesina, on réglait ça à coups de poings!»

Question : "Et il y avait la même solidarité à la Gorgone?»

— E. : «Non, à La Chiesina, on l'appelait la tribu. Il fallait pas toucher quelqu'un de La Chiesina!» (Interview de E. Del Bravo).

La notion de tribu ${ }^{29}$ est ici employée avant tout dans sa dimension territoriale : les habitants de La Chiesina sont une tribu, une organisation territoriale avec un " maire"; une tribu c'est aussi une multitude de personnes qui partagent depuis des générations les mêmes espaces de socialisation. Mais le sens de tribu qui est le plus souligné pour témoigner les sentiments d'estime et de solidarité entre les familles est celui d'unité/opposition : « il fallait pas toucher à quelqu'un de La Chiesina ».

Il n'est pas opportun d'examiner ici les nombreuses problématiques liées à la notion de communauté et à la commémoration nostalgique du passé ; au contraire, il convient de souligner les nuances de lexique dans le discours émique liées à des configurations spécifiques des réseaux matrimoniaux. Dans ce sens, la solidarité «tribale» des habitants de La Chiesina - bien sûr liée à une conception parentale et en accord à la figure du réseau - s'oppose à l' " envie ", au fait de "penser chacun pour soi " des hameaux de la Gorgone, habités par les Marchi et par les Dell'Angelo, et expressions territoriales de la configuration centripète. Cette opposition fondamentale (nous, on s'estime, eux, ils s'envient) est toutefois confortée par les habitants de Gorgone, qui décrivent le lien qui les unit aussi bien en terme de "famille " que d' " envie » :

Question : "Ce groupe [celui des parents et des voisins, décrit plus haut], à quelle occasion agissait-il ?"

— O. : «Jamais, je crois!»

29. Le mot " tribu " était également utilisé pour dénigrer la population de la campagne. Il est intéressant de souligner qu'on le retrouve chez Jolas, Verdier \& Zonabend (1970) pour la France : il désignait un village dans lequel il y avait un grand nombre de mariages entre cousins. 
Question : "Cette parenté, ne servait-elle pas à quelque chose? Quand quelqu'un avait des problèmes d'argent?"

- O.: "Peut-être que je ne m'en souviens pas. Mais je sais que mon père [Dell'Angelo] ne voyait pas d'un très bon oil le frère de sa femme, Pia [Marchi]..., ça ne nous unissait pas, non. Aussi parce que, à la fin, pour moi quelqu'un ne s'est pas bien comporté, parce que, dans la même famille, il faudrait être plus loyal et plus sincère. Mais à la fin, il y en a un qui a roulé la famille, ça ne se fait pas. S'aider dans le sens de donner de l'argent, on ne le faisait pas. Au contraire, ils étaient envieux de ceux [les Marchi ou les Dell'Angelo] qui avaient eu plus de réussite. Ce lien [parental] ne servait pas pour socialiser, pour unir, non, non, non."

Question : "Mais il y a eu des initiatives, comme celle de la société dont vous me parliez..., du vin... Vous souvenez-vous d'autre chose?»

- O. : «Des collaborations du point de vue agricole, mais rien d'autre... Non, autrefois je ne sais pas, mais les derniers temps, il n'y avait pas une bonne entente entre les souches des Dell'Angelo et des Marchi » (Interview de O. Dell'Angelo).

Les familles de La Chiesina étaient en majorité des métayers, mais qui vivaient avec les familles de petits propriétaires, de propriétaires comme les Del Bravo, d'artisans et de charretiers. Le circuit se déroule à travers ces familles, exceptées celles de propriétaires terriens, et passe des métayers aux artisans et parfois aux petits propriétaires. Il est pourtant vrai que ces deux dernières classes professionnelles étaient minoritaires et il est ainsi possible d'affirmer que le circuit représente en général la non-propriété de la famille de métayers, tandis que la configuration centripète traduit, en termes de réseaux, la richesse du petit propriétaire. En poussant l'analyse, il est facile de déduire que l'envie se situe là où il existe un patrimoine à transmettre aux descendants, mais, d'un point de vue éthique, cela paraît trop simpliste. Il est important de souligner, en fait, le résultat à certains égards "paradoxal» qui ressort de la combinaison d'une analyse des graphes et du discours émique. Le graphe à configuration centripète, qui dessine des liens étroitement enchevêtrés, est accompagné des notions de "famille» et d' "envie"; le circuit, au contraire, qui traverse les familles en créant des liens intermédiaires plus que des liens directs, utilise les notions de "tribu " et d' " estime" pour s'exprimer. Les implications théoriques des conclusions de la recherche sont à ce point nombreuses et il est peut-être préférable de considérer ces dernières affirmations comme des hypothèses, afin de développer à l'avenir un dialogue entre la théorie des graphes, l'analyse émique et les notions anthropologiques. 


\section{Dès l'après-guerre, "le monde, tout doucement, commença un peu à se déparenter"}

Le terme de "parenté » indique la consanguinité, la descendance, réelle ou présumée, d'un ancêtre :

- A. : «On est parents, on a le même nom de famille, mais c'est une parenté qui vient de loin, peut-être que les arrière-grands-parents étaient frères. On se respecte! Mais c'est une parenté qui n'existe plus.»

Question : "On se respecte, dans quel sens?»

— A. : "Comme on porte le même nom... Et que mon grand-père et le grand-père de Mino étaient peut-être cousins au troisième degré. Même s'il n'y a plus de parenté, on se respecte, on est amis, on porte le même nom... c'est comme si on était..."

— R. : «... des parents proches» (Interview de An. Dell'Angelo et A. Ricci).

On a introduit le terme "parenté " pour mieux réfléchir à son contraire, c'est-à-dire au terme sparentamento, " déparenté ». La perception actuelle des habitants en ce qui concerne le choix du conjoint est celle d'un passage des réseaux parentaux aux réseaux amicaux : sur le marché matrimonial actuel les parents des parents sont remplacés par les amis des amis, qui permettent d'atteindre des candidats potentiels au mariage autrement trop éloignés. Ce phénomène d'un réseau social embrassant tout, qui peut être en partie exploré grâce à des liens intermédiaires, était apparu dans l'analyse démographique du marché matrimonial de Lucignano, où ressortaient des lieux privilégiés de choix du conjoint comme Cortona ou Castiglion Fiorentino pour l'exogamie masculine, et Rapolano pour l'exogamie féminine :

Question : «Et vous, comment avez-vous fait connaissance ? Parce que, lorsque j’ai élaboré les données que j'avais sur les actes de mariage, j'ai pu constater que, à partir de 1960, Cortona [lieu d'origine de la femme de celui que j'interviewe] devient une ville où beaucoup d'habitants de Lucignano trouvent une épouse. »

- M. : "Comme à Castiglion Fiorentino! Par vagues, oui. Mais je crois que c'est le hasard, parce qu'à Castiglion Fiorentino, il pouvait se faire qu'on rencontre une fille, et puis après... Avant d'arriver à Cortona, j'ai été à Castiglioni. On allait avec des copains danser à Castiglioni, c'est sûr que si l'un d'entre nous rencontrait des filles, il nous les présentait, et elles, elles nous présentaient des amis, et ainsi de suite. Moi, j’en ai connus au moins trois, quatre... [mariages se concluant de cette façon]. Mais nous à Cortona, on est plus chacun pour soi. Moi, j'ai connu ma femme parce que j'avais retrouvé un ancien copain d'école de son village, qui en plus a été notre témoin de noces, et il m'a raconté qu'ils préparaient une fête, la fête des chariots agricoles... il m'a invité à cette fête. Le samedi soir, il y avait une pièce de théâtre... ça m'intéressait, moi et un autre copain, qui fréquentait la sœur de ma femme. Alors je me suis souvenu de l'invitation et je me suis dit: "Pourquoi pas, on pourra discuter un peu". Après, il m’a présenté des filles, et elle, elle y était. Mais c'était le hasard, parce que ce qui m’intéressait, c'était de voir la pièce de théâtre " (Interview de M. Ter. et De P., qui se sont mariés en 1975). 
L'exploration des réseaux informels des amis, ou des amis des amis, permet à un candidat au mariage de trouver un partenaire avec lequel il n'a aucun contact mais des amis en commun, tout comme autrefois les deux époux partageaient un parent dans certains réseaux matrimoniaux. Cette transformation que le marché matrimonial manifeste dans sa façon de procéder, en partant des réseaux parentaux jusqu'aux réseaux amicaux, mériterait d'être approfondie en termes de configuration, de contenu et de sens. Il serait intéressant surtout d'en comprendre les implications sur la notion de "personne " et de valeur matrimoniale; on aimerait conclure, en effet, que la personne, là où autrefois elle montrait sa famille, montre aujourd'hui ses amis. Il est assez difficile de situer ce changement à un moment temporel précis. On dit que dès l'après-guerre «le monde, tout doucement, commença un peu à "se déparenter" (sparentissi)». Cette expression est une magnifique représentation du réseau parental, observé au moment même où il est occupé à agrandir ses maillons afin de permettre à d'autres types de liens, comme l'amitié, d'avoir un poids plus important dans les dynamiques de choix du conjoint.

La «déparenté » du monde (sparentamento) renvoie essentiellement à la chute de la natalité, à la réduction des liens collatéraux, à la dispersion résidentielle des membres de la famille et des patronymes, d'où la diminution des bassins matrimoniaux, expressions de réseaux denses de parents et de parents des parents. La «déparenté » du monde «brouille les arbres généalogiques» (Deleuze \& Guattari 1980) et les fait mourir.

Università di Siena, Italie sinibaldi@unisi.it

MOTS CLÉS/KEYWORDS : réseaux matrimoniaux/marriage networks - théorie des graphes/social network analysis - mémoire familiale/family memory - patrimoine/patrimony - parenté européennel european kinship - Toscane/Tuscany. 
Augustins, Georges

1989 Comment se perpétuer? Devenir des lignées et destins des patrimoines dans les paysanneries européennes. Nanterre, Société d'ethnologie.

\section{Becker, Gary S.}

1981 A Treatise on the Family. CambridgeLondon, Harvard University Press.

1998 L'Approccio economico al comportamento umano. Bologna, Il Mulino.

Bertin, Jacques

1999 [1967] Sémiologie graphique: les diagrammes, les réseaux, les cartes. Paris, Éd. de l'Ehess.

\section{Bourdieu, Pierre}

1989 "Reproduction interdite : la dimension symbolique de la domination économique ", Études rurales 113-114 : 15-36. 1994 Raisons pratiques: sur la théorie de l'action. Paris, Le Seuil.

\section{Brunet, Guy, Antoinette Fauve-Chamoux} \& Michel Oris, eds

1998 Le Choix du conjoint: premiers Entretiens de la Société de démographie historique, Paris, 15-16 novembre 1996. Villeurbanne, Programme Rhône-Alpes, Recherche en sciences humaines.

\section{Caillé, Alain}

1994 Don, intérêt et désintéressement: Bourdieu, Mauss, Platon et quelques autres. Paris, La Découverte - MAUSS.

\section{Deleuze, Gilles \& Félix Guattari}

1980 Mille plateaux : capitalisme et schizophrénie. Paris, Minuit.

\footnotetext{
De Nooy, Wouter, Andrej Mrvar \& Vladimir Batagelj

2004 Exploratory Social Network Analysis with Pajek [http ://vlado.fmf.uni-lj.si/ pub/networks/pajek/].
}

Halbwachs, Maurice

1994 [1925] Les Cadres sociaux de la mémoire. Paris, Albin Michel.

Héritier-Augé, Françoise \& Élisabeth CopetRougier, eds

1991 Les Complexités de l'alliance. 2, Les systèmes complexes d'alliance matrimoniale. Paris, Éd. des Archives contemporaines.

Houseman, Michael \& Douglas R. White 1996 «Structures réticulaires de la pratique matrimoniale », L'Homme 139 : 59-86.

Jolas, Tina, Yvonne Verdier \& Françoise Zonabend

1970 "Parler famille », L'Homme 10 : 5-26.

Pina Cabral, João de

1989 "L'héritage de Maine : repenser les catégories descriptives dans l'étude de la famille en Europe ", Ethnologie française 19 (4) : 329-340.

Sinibaldi, Silvia

2002 «Lucignano come centro : l'area alfa $1 »$, in Pier Giorgio Solinas \& Simonetta Grilli, Spazi di alleanza: aree di matrimonialità nella Toscana meridionale. Roma, CISU : 189-218.

2002-2003 Valore dei beni e valori della persona: la scelta del coniuge in un comune della Val di Chiana nel corso del XX secolo. Siena, Università degli studi di Siena, thèse de doctorat.

2004 "Com'è la vite ci metti 'u palo : la mediazione matrimoniale tra toscani e donne calabresi (1950-1990)", in Bruno Wanrooij, ed., La Mediazione matrimoniale : il terzo (in)comodo in Europa fra Otto e Novecento. Fiesole-Roma, Edizioni di Storia e Letteratura : 221-253. 


\section{Solinas, Pier Giorgio}

1987 «La dot et la part: transmission des biens, fils et filles dans les familles polynucléaires des métayers siennois ", in Georges Ravis-Giordani, ed., Femmes et patrimoine dans les sociétés rurales de l'Europe méditerranéenne. Paris, Éd. du Cnrs : 169-188.

1992 "La residenza instabile", La Ricerca folklorica 25 : 47-50.
Solinas, Pier Giorgio \& Simonetta Grilli

2002 Spazi di alleanza: aree di matrimonialità nella Toscana meridionale. Roma,

CISU.

Wasserman, Stanley \& Katherine Faust

1994 Social Network Analysis: Methods and Applications. Cambridge-New York, Cambridge University Press.

\section{RÉSUMÉ/ABSTRACT}

Silvia Sinibaldi, Réseaux des liens matrimoniaux: le choix du conjoint dans une commune toscane au $X X^{e}$ siècle. - Lucignano in Val di Chiana est un bourg de la Toscane du Sud (Italie), qui a fait l'objet de cette recherche historique, anthropologique et démographique concernant la nuptialité, les formes de famille et les réseaux de parenté au cours du $\mathrm{XX}^{\mathrm{e}}$ siècle. L'exploration des dynamiques de choix du conjoint a nécessité une méthode ethnographique composite, pouvant conjuguer des plans de connaissance différents (quantitatif, morphologique, subjectif, symbolique). Dans l'article, l'auteur examine les morphologies des réseaux des liens matrimoniaux de groupes socioprofessionnels déterminés : les configurations stellaires des grands propriétaires, les renchainnements (graphe à structure centripète) des petits propriétaires, les circuits dans les réseaux des métayers. Ces trois "paradigmes morphologiques " s'expriment, dans le discours émique, par une originale combinaison des termes de "famille" et "tribu ", "parenté" et "déparenté" (sparentamento), " estime " et « envie».
Silvia Sinibaldi, Networks of Matrimonial Bonds : Choosing a Spouse in a Tuscan Town in the 20th Century. - Lucignano, a town in Val di Chiana (southern Tuscany, Italy), is the site of this historical, anthropological and demographic study of marriage, family forms and kinship networks in the 20th century. A composite method combining various types of knowledge (quantitative, morphological, subjective, symbolic) had to be used to explore the dynamics behind the choice of a spouse. The morphology of networks of matrimonial bonds in specific socioeconomic groups is presented: the star-like patterns of big landowners, the "relinking" (a centripetal graph) of small landowners and " circuits" among sharecroppers. These three patterns are expressed in emic discourse through an original combination of the words "family " and " tribe ", " kinship " and " unkinship " (sparentamento), " esteem " and " envy". 